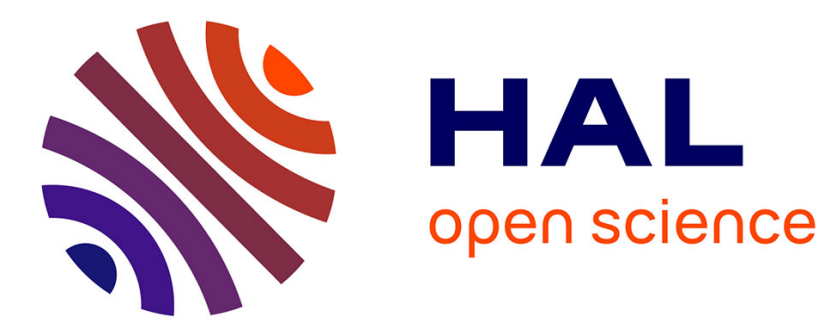

\title{
Local field potentials in primate motor cortex encode grasp kinetic parameters
}

Tomislav Milekovic, Wilson Truccolo, Sonja Grün, Alexa Riehle, Thomas Brochier

\section{> To cite this version:}

Tomislav Milekovic, Wilson Truccolo, Sonja Grün, Alexa Riehle, Thomas Brochier. Local field potentials in primate motor cortex encode grasp kinetic parameters. NeuroImage, 2015, 114, pp.338 - 355. 10.1016/j.neuroimage.2015.04.008 . hal-01464171

\section{HAL Id: hal-01464171 https://hal-amu.archives-ouvertes.fr/hal-01464171}

Submitted on 16 Feb 2017

HAL is a multi-disciplinary open access archive for the deposit and dissemination of scientific research documents, whether they are published or not. The documents may come from teaching and research institutions in France or abroad, or from public or private research centers.
L'archive ouverte pluridisciplinaire HAL, est destinée au dépôt et à la diffusion de documents scientifiques de niveau recherche, publiés ou non, émanant des établissements d'enseignement et de recherche français ou étrangers, des laboratoires publics ou privés. 


\section{Local field potentials in primate motor cortex encode grasp kinetic parameters}

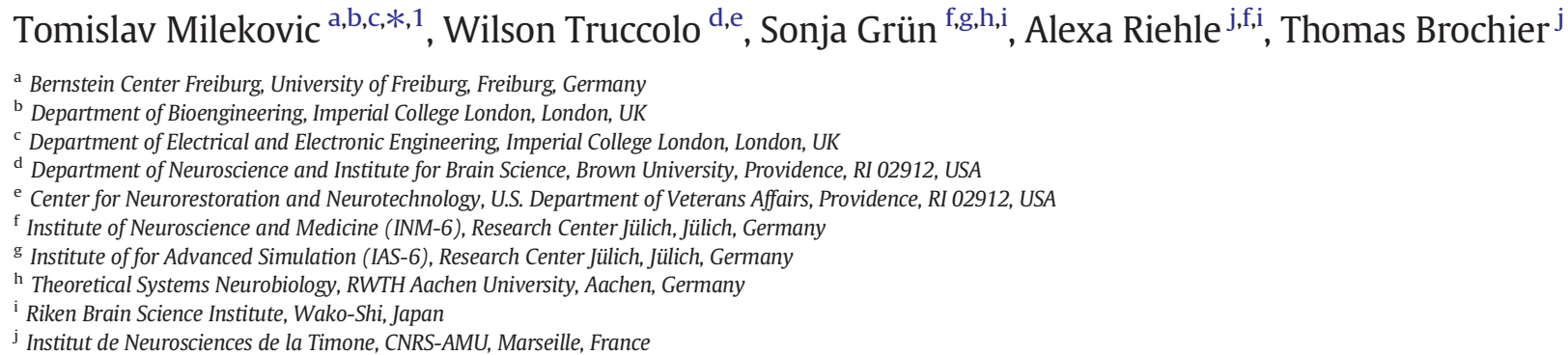

\section{A R T I C L E I N F O}

\section{Article history:}

Received 3 December 2014

Accepted 2 April 2015

Available online 11 April 2015

\section{Keywords:}

Macaque

Motor cortex

Kinetics

Grasping

Local field potentials

Movement planning

\begin{abstract}
A B S T R A C T
Reach and grasp kinematics are known to be encoded in the spiking activity of neuronal ensembles and in local field potentials (LFPs) recorded from primate motor cortex during movement planning and execution. However, little is known, especially in LFPs, about the encoding of kinetic parameters, such as forces exerted on the object during the same actions. We implanted two monkeys with microelectrode arrays in the motor cortical areas MI and PMd to investigate encoding of grasp-related parameters in motor cortical LFPs during planning and execution of reach-and-grasp movements. We identified three components of the LFP that modulated during grasps corresponding to low $(0.3-7 \mathrm{~Hz})$, intermediate $(\sim 10-\sim 40 \mathrm{~Hz})$ and high $(\sim 80-250 \mathrm{~Hz})$ frequency bands. We show that all three components can be used to classify not only grip types but also object loads during planning and execution of a grasping movement. In addition, we demonstrate that all three components recorded during planning or execution can be used to continuously decode finger pressure forces and hand position related to the grasping movement. Low and high frequency components provide similar classification and decoding accuracies, which were substantially higher than those obtained from the intermediate frequency component. Our results demonstrate that intended reach and grasp kinetic parameters are encoded in multiple LFP bands during both movement planning and execution. These findings also suggest that the LFP is a reliable signal for the control of parameters related to object load and applied pressure forces in brain-machine interfaces.
\end{abstract}

(c) 2015 Elsevier Inc. All rights reserved.

\section{Introduction}

Advances in chronically implanted intracortical multielectrode technology pave the way in understanding the function of motor cortex in complex upper limb control. Neural recordings obtained from microelectrode arrays contain action potentials (spikes) of an ensemble of neurons as well as local field potentials (LFPs), which are thought to represent a population measure that mainly reflects the local synaptic

\footnotetext{
* Corresponding author at: Center for Neuroprosthetics and Brain Mind Institute, School of Life Sciences, Swiss Federal Institute of Technology (EPFL), EPFL SV UPCOURTINE, Station-19, 1015 Lausanne, Switzerland. Fax: + 410216937270

E-mail addresses: tomislav.milekovic@epfl.ch (T. Milekovic),

wilson_truccolo@brown.edu (W.Truccolo),s.gruen@fz-juelich.de (S. Grün), alexa.riehle@univ-amu.fr (A. Riehle), thomas.brochier@univ-amu.fr (T. Brochier).

${ }^{1}$ Current address the same as the corresponding address.
}

activity, with contributions from spike after-potentials and intrinsic trans-membrane current changes in the vicinity of the recording electrodes (Buzsaki et al., 2012; Logothetis et al., 2007; Mitzdorf, 1985; Reimann et al., 2013; Waldert et al., 2013). Large number of electrodes and high sampling rates in microelectrode arrays offer the opportunity to investigate the temporal evolution of stimulus and behavior-related information encoded in the recorded neural signals and, thus, estimate the function of the implanted cortical area.

Recently, it has been shown that hand and finger kinematics are accurately encoded in the spiking activity of motor cortical neurons (Bansal et al., 2012; Saleh et al., 2012; Vargas-Irwin et al., 2010). It has also been shown that spiking activity of motor cortical neurons encodes different grip types (Mollazadeh et al., 2011). Furthermore, it has been reported that movement direction and different grip types can be reliably classified from the modulation of single unit activities preceding movement execution (Carpaneto et al., 2011; Santhanam et al., 2006; 
Townsend et al., 2011). Modulation of motor cortical firing rates related to grip aperture, grip type and grip force preceding and during grasp movements was also demonstrated (Hendrix et al., 2009).

Previous studies reported that the LFP also encodes movement kinematics, such as reaching direction (Flint et al., 2012b; Mehring et al., 2003; Rickert et al., 2005), grip types (Li et al., 2012; Mollazadeh et al., 2011), hand and finger kinematics (Bansal et al., 2012) as well as arm muscle activation during reach-and-grasp movements (Flint et al., 2012a). However, the presence of grasp-related information, in particular kinetic parameters during both movement planning and execution, has not yet been demonstrated in LFP signals recorded from motor cortical areas. In particular, the understanding of the temporal dynamics of grasp encoding in motor cortical LFPs is still lacking.

Several recent studies examined grasp-related information in motor cortical areas by investigating recordings of cortical surface potentials (electrocorticography; ECoG) in monkeys (Chen et al., 2014) and humans (Flint et al., 2014; Pistohl et al., 2012). These studies demonstrated that ECoG accurately encodes different grasp types, finger pressure forces and activity of finger muscles during the grasp execution. ECoG is thought to represent summed postsynaptic potentials originating at the cortical surface (Miller, 2010; Miller et al., 2009). Deeper cortical layers perform computation and their activity, thus, may differ from that at the surface (Leski et al., 2013). The spatial resolution of LFPs recorded with microelectrodes is adequate to analyze signals originating from deeper cortical layers without interference from surface potentials (Leski et al., 2013; Xing et al., 2009), as shown by different amounts of behavior-related information present in the LFPs recorded at different cortical depths (Markowitz et al., 2011). On the other hand, the ECoG spatial resolution is substantially lower than that of LFPs recorded with microelectrodes, which can lead to lower signalto-noise ratio of signals originating from sparsely distributed sources. Several studies suggested that finger force representations may indeed be distributed sparsely throughout the motor cortical areas (Flint et al., 2014; Kubanek et al., 2009; Schieber and Poliakov, 1998). Furthermore, to our knowledge, a systematic assessment of force information as well as the time course of kinetic information during both preparation and execution of reach/grasp actions has not been done previously. For all these reasons, an investigation of grasp-related information collected from LFPs recorded from deeper cortical layers may provide an important advance with respect to the above mentioned previous studies.

Here, we analyzed LFP signals recorded from the motor cortex of two monkeys performing an instructed delayed reach-to-grasp task. LFP modulations were investigated in relation to two task parameters: (i) the grip type used to grasp the object and (ii) the object load. We also recorded pressure forces of thumb, index finger and middle finger while the monkey was holding the object. We demonstrate that, by using low (0.3-7 Hz bandpass filtered signal) and high (spectral amplitude in $\sim 80-250 \mathrm{~Hz}$ band) frequency components of the LFPs, grip type and loading force can be classified with different levels of accuracy long before movement initiation. Additionally, we show that intended finger pressure forces applied on the object during object movement can be reliably decoded from LFP signals recorded both during the delay period of the trial and during the execution of the grasp movement. Finally, using a time-resolved analysis of decoding, we mapped the temporal evolution of the grasp-related information encoded in motor cortical LFPs.

\section{Methods}

\section{Behavioral task}

Two female macaque monkeys ( $\mathrm{L}$ and T, 4.5 and $5.5 \mathrm{~kg}$ ) performed an instructed and delayed object reach, grasp and pull task previously described in Riehle et al. (2013) and summarized in Fig. 1a and b. Monkey L performed the task with her left hand and monkey $\mathrm{T}$ with her right hand. The target object was a stainless steel parallelepiped ( $40 \mathrm{~mm} \times 16 \mathrm{~mm} \times 10 \mathrm{~mm}$ ) mounted on a horizontal shuttle and rotated at a $45^{\circ}$ angle from the vertical axis. The object was located about $20 \mathrm{~cm}$ away from the monkey. Monkeys were instructed to grasp the object using one of two distinct grips: (i) a precision grip (PG) or (ii) a side grip (SG; Fig. 1). In PG, they placed the tips of the index and the thumb in a groove on the upper and lower sides of the object, respectively. In PGs, the monkeys also placed the lateral side of the middle finger in contact with the left (monkey L) or the right (monkey T) side of the object. In SG, they placed the thumb and the lateral side of the middle finger on the opposite sides of the object while placing the index finger in the upper groove (monkey L: thumb - right; middle finger - left; monkey T: thumb - left; middle finger - right; Fig. 1c). The monkeys pulled the object towards their bodies against a high force (HF) or a low force (LF). LF and HF were imposed by a weight connected to the back side of the object (object load) hidden from the monkeys and were roughly $0.6 \mathrm{~N}$ and $1.6 \mathrm{~N}$ for monkey $\mathrm{T}$ and $1 \mathrm{~N}$ and $2 \mathrm{~N}$ for monkey L. Changes in weights between trials were computer controlled and were occluded from the monkeys' view. The detection of correct grip types was performed online by controlling that the pressure force applied by the thumb exceeded a $0.2 \mathrm{~N}$ threshold on a predefined sensor of the object. Thumb pressure force had to exceed the threshold on the bottom sensor in PG trials for both monkeys; and on the right or left sensor in SG trials for monkey L and T, respectively. Visual inspection during training sessions demonstrated that each monkey adopted a stereotyped strategy to grasp the object and that these criteria were highly reliable to classify between precision grip and side grip trials during performance of the task. In addition, monkeys were video monitored during all recording sessions to ensure that they always used the same position of the fingers in respect to the object. Force sensing resistor (FSR) covered each side of the object and were in turn covered by thin metal plates on which the monkey placed his fingers (Supplementary Fig. 1). Thin hemispheric plastic pads, $5 \mathrm{~mm}$ in diameter, were glued bellow the plate to transfer any force applied on the plate in a force applied at a single contact point on the FSR. This design provided a continuous measure of the pressure forces on each side of the object. In addition, a hall-effect sensor measured the horizontal displacement of the object over a maximal distance of $15 \mathrm{~mm}$. The light in the room was dimmed and monkeys could see the object during the execution of the task. A square of 4 red light-emitting diodes (LEDs) with one yellow LED in the center was used to display the task instructions. To initiate a trial, monkeys had to press the home switch, positioned at waistlevel, with their trained hand. After $400 \mathrm{~ms}$, the yellow LED was turned on to mark the trial onset. Following another $400 \mathrm{~ms}$, an informative cue, in the following called "CUE", was presented, disclosing either the grip type (grip cue task; GRIP) or the object load (force cue task; FORCE). The cue was given by illuminating two out of four LEDs. The meaning of the cue was as follows: (i) the two bottom LEDs for LF, (ii) the two top LEDs for HF, (iii) the two left LEDs for SG, and (iv) the two right LEDs for PG. The cue was presented for $300 \mathrm{~ms}$ and was followed by a $1 \mathrm{~s}$ preparatory delay period. At the end of the delay period, the go signal, in the following called "GO", provided the remaining information either about the force (in GRIP) or the grip (in FORCE) by illuminating the appropriate combination of LEDs. GRIP and FORCE conditions were tested in separate "sessions", here defined as periods during which monkeys performed the task without a pause. Thus, GRIP sessions consisted of SG/HF, SG/LF, PG/HF and PG/LF trials, while FORCE sessions consisted of HF/SG, LF/SG, HF/PG and LF/PG trials. The different trial types were presented in a randomized order. GO also served as imperative signal instructing the monkeys to release the switch and to reach and grasp the object and pull it towards them. We refer to the start of the object manipulating movement as "object movement onset". Monkeys were rewarded with a drop of mixture composed of $50 \%$ apple sauce and $50 \%$ water. To receive the reward, the monkeys had to release the switch within $1 \mathrm{~s}$ after the GO, grasp the object with their trained hand using the instructed grasp type, pull the object 

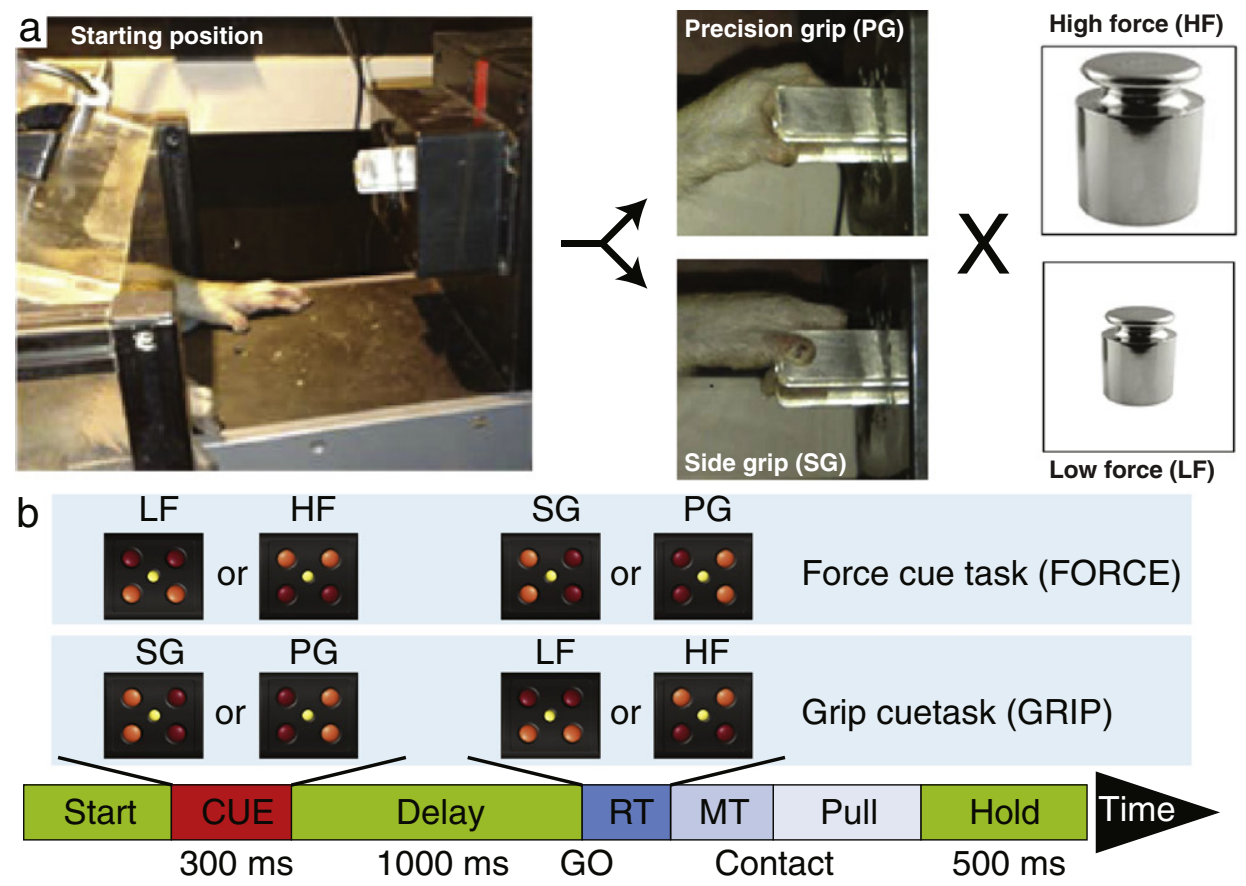

C
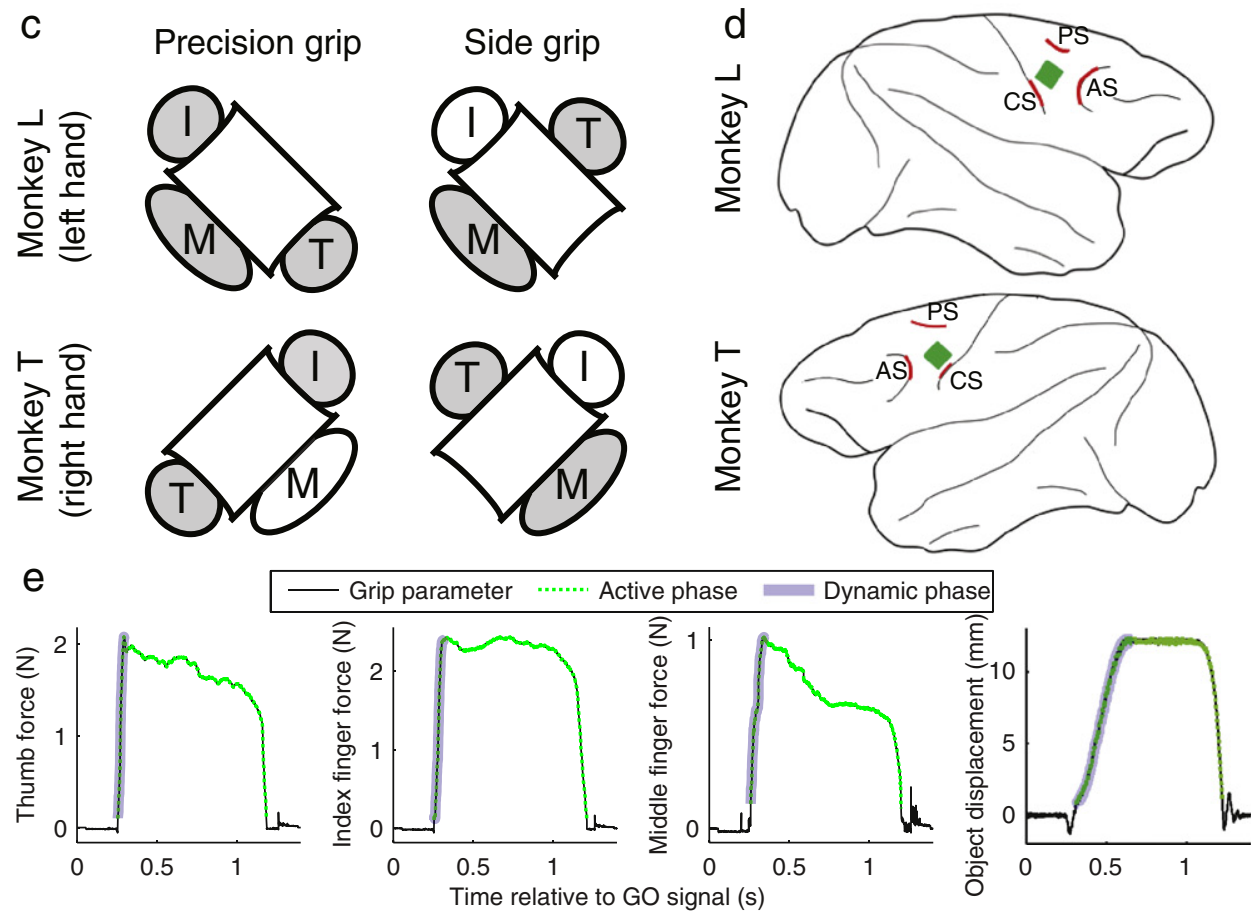

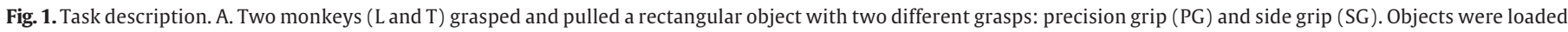

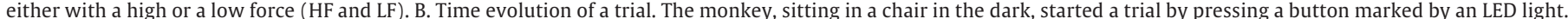

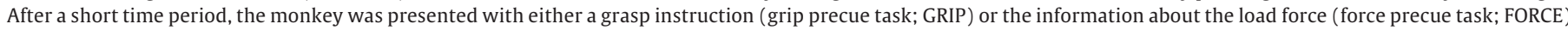

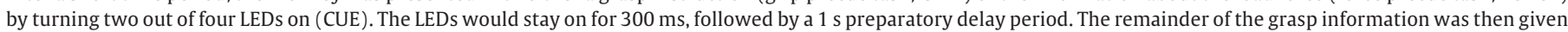

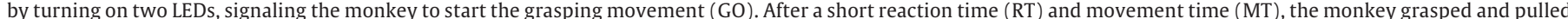

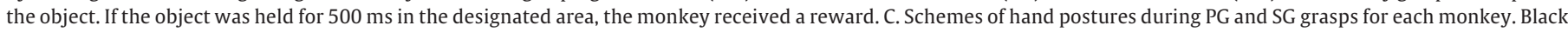

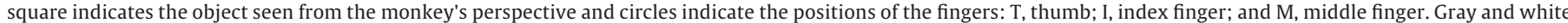

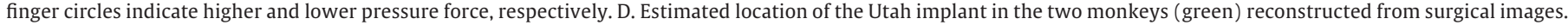

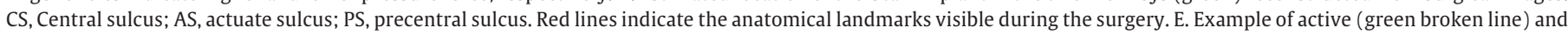
dynamic (blue tube) phases of grip parameters during one PG/HF trial for monkey L.

towards them until it reaches the distance between $4 \mathrm{~mm}$ and $14 \mathrm{~mm}$ and hold the object within that range for $500 \mathrm{~ms}$. The distance limits were removed in the FORCE condition in which the monkeys had more difficulty to perform the task. To start a new trial, the monkeys had to return their trained hand to the starting position and press the home switch. All animal procedures were approved by the local ethical committee (authorization A1/10/12) and conformed to the European and French government regulations. 


\section{Surgery and data acquisition}

Monkeys were trained for about a year until they achieved the task with at least $80 \%$ success rate. Once the monkeys were trained, a 100 electrodes Utah array (Blackrock Microsystems, Salt Lake City, UT, USA) was surgically implanted in the motor cortex contralaterally to the trained hand (right hemisphere for monkey L and left hemisphere for monkey T). The array had an arrangement of $10 \times 10$ Iridium Oxide electrodes, each of them $1.5 \mathrm{~mm}$ long, with an inter-electrode distance of $400 \mu \mathrm{m}$. We assume that recordings obtained with this electrode length originate from layer 5 . The surgery was performed under deep general anesthesia using full aseptic procedures. Anesthesia was induced with $10 \mathrm{mg} / \mathrm{kg}$ i.m. ketamine and maintained with $2-2.5 \%$ isoflurane in 40:60 $\mathrm{O}_{2}$-air. To prevent cortical swelling, $2 \mathrm{ml} / \mathrm{kg}$ of mannitol was slowly injected i.v. over a period of $10 \mathrm{~min}$. A $20 \times 20 \mathrm{~mm}$ craniotomy was performed over the motor cortex and the dura was incised and reflected. Based on anatomical landmarks visible during the surgery, the array was positioned on the cortical surface $3 \mathrm{~mm}$ anterior to the central sulcus at the level of the spur of the arcuate sulcus (Fig. 1d; for surgical photos see Supplementary Fig. 2). The array was inserted using a pneumatic inserter (Array Inserter, Blackrock Microsystems) and covered with a sheet of an artificial, non-absorbable dura (Goretex). The real dura was sutured back and covered with a piece of an artificial absorbable dura (Seamdura, Codman). The bone flap was put back at its original position and attached to the skull by means of a $4 \times 40 \mathrm{~mm}$ strip of titanium (Bioplate, Codman). The array connector was fixed to the skull on the opposite side with titanium bone screws (Bioplate, Codman). The skin was sutured back over the bone flap and around the connector. The monkey received a full course of antibiotics and analgesic during the week following the surgery.

Data recording and storage were made using a 128-channel data acquisition system (Cerebus, Blackrock Microsystems). The signal from each active electrode ( 96 out of the 100 electrodes were connected) was pre-processed (analog band-pass filtered, filter cutoff frequencies: $0.3 \mathrm{~Hz}-7.5 \mathrm{kHz}$, sampled at $30 \mathrm{kHz}$ ) by a head stage with unit gain and then amplified with a gain of 5000. The signal was then filtered in two different frequency bands in order to split it into local field potentials (LFPs, filter cutoff frequencies: $0.3-250 \mathrm{~Hz}$ ) and spiking activity (filter cutoff frequencies: $0.5-7.5 \mathrm{kHz}$ ). The LFPs were down-sampled to $1 \mathrm{kHz}$ and saved on disk. During each behavioral session, grip forces and object displacement were recorded with a $1 \mathrm{kHz}$ sampling rate and saved along with the neural recordings and task events (CUE onset and offset, GO, switch release, reward). Times of the object movement onset were identified by taking the first time point after GO for which the object displacement crossed $1.25 \mathrm{~mm}$ for monkey L and $1.5 \mathrm{~mm}$ for monkey $\mathrm{T}$ from the initial position of the object.

During the manipulation of the object by the monkey, pressure forces applied by her thumb, index finger and middle finger were measured by the force plates on the sides of the object. While the monkey held the object, the position of her trained hand was affine related to the measured object displacement. However, while a finger was not in contact with the object or while the object was not moved, the corresponding grip parameter, i.e. one of thumb, index finger or middle finger pressure force or object displacement, could not be determined. Therefore, we defined the "active" phase of each trial as the time period after GO for which the value of the grip parameter was above the parameter-specific threshold (Table 1). The thresholds were selected

Table 1

Thresholds used to identify active phase for each grip parameter in each trial.

\begin{tabular}{lll}
\hline Grip parameter & Monkey L & Monkey T \\
\hline Thumb force & $0.07 \mathrm{~N}$ & $0.05 \mathrm{~N}$ \\
Index finger force & $0.05 \mathrm{~N}$ & $0.04 \mathrm{~N}$ \\
Middle finger force & $0.1 \mathrm{~N}$ & $0.05 \mathrm{~N}$ \\
Object displacement & $0.75 \mathrm{~mm}$ & $1.25 \mathrm{~mm}$ \\
\hline
\end{tabular}

upon visual inspection of the grip parameter traces. In addition, within the active phase, we defined the "dynamic" phase of the trial as the period in which grip parameters were increasing. The beginning of the dynamic phase was the same as the beginning of the active phase and the end was the first moment at which a grip parameter stopped increasing. This moment was determined as the first zero crossing of the derivative of the grip parameter, as calculated by the 1st derivative Savitzky-Golay filter (3rd order, $61 \mathrm{~ms}$ long window). See Fig. 1e for an example trial with active and dynamic phases marked.

\section{Data processing}

Only LFPs were analyzed in this study. For each monkey, we selected a number of sessions that satisfied the following criteria: (i) grip parameter recordings contained only a small number of mechanical artifacts, and (ii) sessions of the same type (GRIP or FORCE) recorded on three consecutive days contained at least 400 trials. The latter criterion was used to generate a larger set of data during which the encoding of behavior in neural activity would remain stationary. Same type sessions recorded over three consecutive days were then grouped into one dataset. For each monkey, we formed two datasets in the GRIP task (LGRIP1, L-GRIP2 and T-GRIP1, T-GRIP2 for monkey L and T, respectively) and one dataset in the FORCE task (L-FORCE1 and T-FORCE1 for monkey $\mathrm{L}$ and $\mathrm{T}$, respectively; see Table 2 ).

\section{Processing of behavioral responses}

In the majority of trials, the duration between the GO and the object movement onset was less than $1 \mathrm{~s}$. This duration combines the reaction time (RT) between the GO until switch release and the movement time (MT) from switch release until object movement onset. To ensure homogeneous timing for these phases of the trial and to accumulate stereotypical LFP responses, we identified trials where RT + MT exceeded $1 \mathrm{~s}$ and removed them from further analysis (see Table 2 for the number of trials removed from each dataset). See Fig. 2 for mean traces of grip parameters for each dataset.

We investigated the extent to which grasp types and load forces had an effect on the grip parameters executed by the monkeys. To this end, we calculated the mean grip parameter during the active phase of the trial $\left(\mu_{G P}\right)$ and modeled it as a linear function of the grip type and load force.

$\mu_{G P}(t r)=a_{0}+a_{G} \cdot G(t r)+a_{F} \cdot F(t r)$

where $t r$ is the trial number; $G$ is variable describing the grip type and is 0 for SG and 1 for PG; $F$ is the variable describing the load force and is 0 for LF and 1 for $\mathrm{HF}$; and $a_{0}, a_{G}$ and $a_{F}$ are coefficients of the linear fit. The coefficients were calculated separately for GRIP and FORCE dataset for each monkey. To verify that the linear model explained large portion of the variance, we also measured the $r^{2}$ of the fit.

\section{Processing of neural recordings}

Within each dataset, from the set of 96 LFP channels, we identified a subset of "reference" channels ( ref) that contained a small amount of line

Table 2

List of sessions performed by each monkey. RT - reaction time; MT - movement time.

\begin{tabular}{lllll}
\hline Dataset & $\begin{array}{l}\text { Total number of sessions } \\
\text { (in each day) }\end{array}$ & $\begin{array}{l}\text { Total } \\
\text { successful } \\
\text { trials }\end{array}$ & $\begin{array}{l}\text { MT }+ \text { RT } \\
>1 \mathrm{~s}\end{array}$ & $\begin{array}{l}\text { Trials after } \\
\text { removal }\end{array}$ \\
\hline L-GRIP1 & $7(2,3,2)$ & 933 & 0 & 933 \\
L-GRIP2 & $5(3,1,1)$ & 630 & 4 & 626 \\
T-GRIP1 & $4(2,2)$ & 507 & 49 & 458 \\
T-GRIP2 & $4(1,3)$ & 516 & 13 & 503 \\
L-FORCE1 & $4(2,0,2)$ & 506 & 15 & 491 \\
T-FORCE1 & $4(2,1,1)$ & 487 & 59 & 428
\end{tabular}




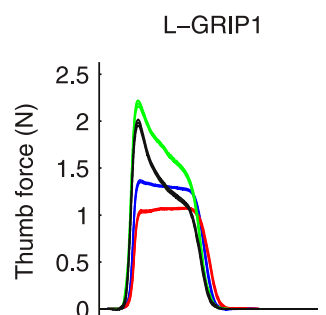

$$
\text { L-GRIP2 }
$$

L-FORCE1
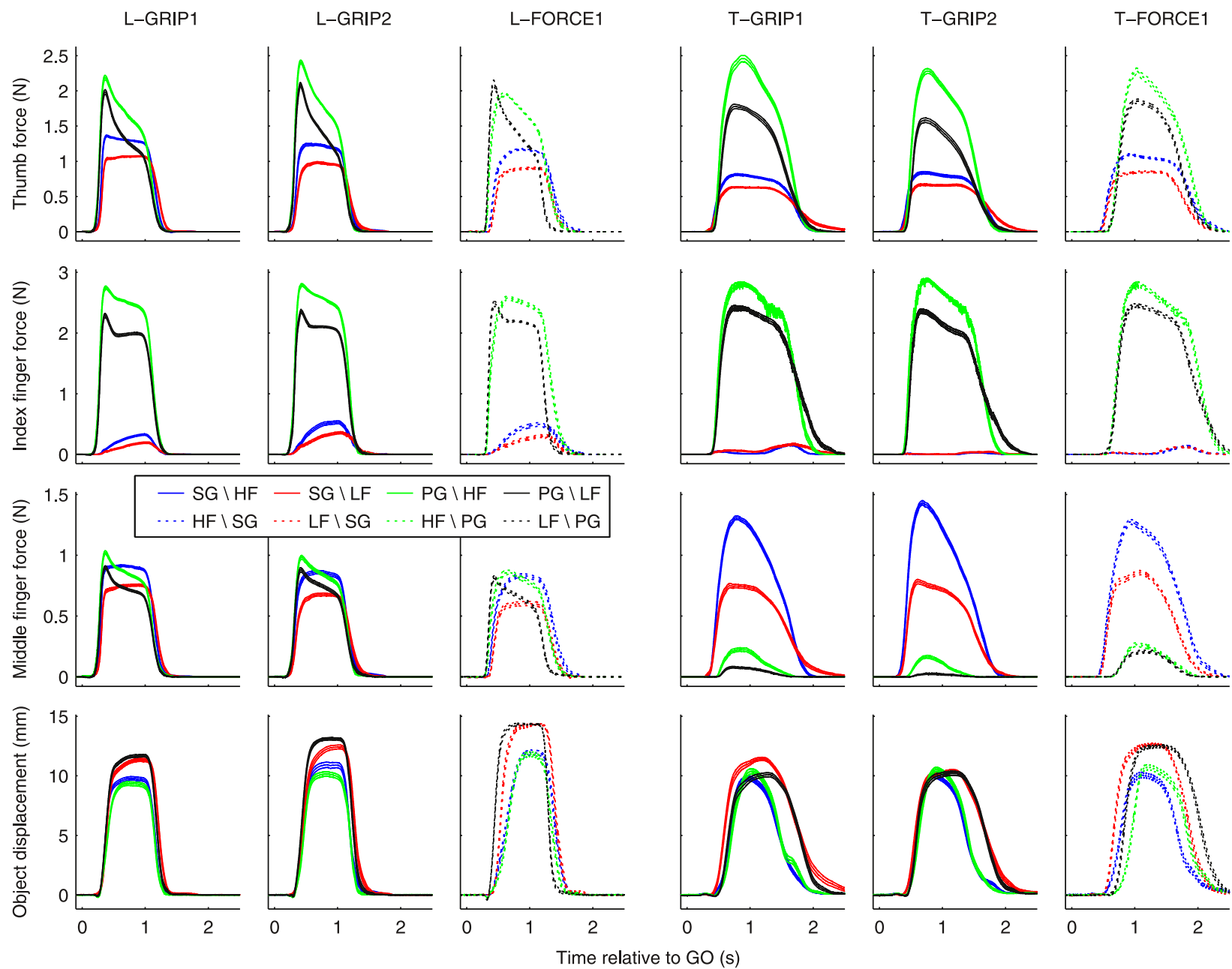

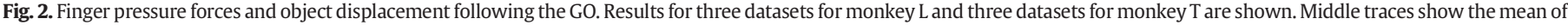

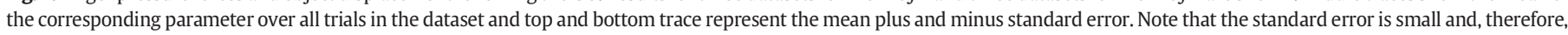
the three lines are close together and may not be easily distinguishable. SG - side grip; PG - precision grip; LF - low force; HF - high force.

noise $(50 \mathrm{~Hz})$ and no obvious recording artifacts (number of reference channels identified: L-GRIP1: 83; L-GRIP2: 71; L-FORCE1: 69; T-GRIP1: 31; T-GRIP2: 29; T-FORCE1: 30). We defined a common average reference channel $\Phi_{C A R}$ as the mean over all channels in the ref subset.

$\Phi_{C A R}(t)=\frac{1}{N_{\text {ref }}} \sum_{c h_{\text {ref }}} \Phi\left(c h_{\text {ref }}, t\right) \quad c h_{r e f} \in$ ref

where $N_{\text {ref }}$ is the number of channels in the ref subset and $\Phi\left(c h_{r e f}, t\right)$ is the LFP recorded on reference channel $c h_{\text {ref }}$ at time $t$. To reference the LFPs to the common average $(\bar{\Phi})$, i.e. to remove the common average, we subtracted $\Phi_{C A R}$ from all channels in the ref subset and used those in the remainder of the analysis.

$\bar{\Phi}\left(c h_{r e f}, t\right)=\Phi\left(c h_{r e f}, t\right)-\Phi_{C A R}(t) \quad c h_{r e f} \in$ ref.

Referencing to common average makes the ref subset channels linearly dependent (see Supplementary materials for demonstration). To restore the linear independence, the first channel of the ref subset was excluded from further analysis. Thus, the rest of the analysis was performed on a ref' channel subset defined as:

$r e f^{\prime}=\operatorname{ref} c h_{r e f}(1) \quad c h_{r e f}^{\prime} \in r e f^{\prime}$ where $c h^{\prime}{ }_{\text {ref }}$ are the members of the ref ' subset and $N^{\prime}{ }_{\text {ref }}=N_{\text {ref }}-1$ is the number of channels in the ref' subset.

\section{Identifying neural response components}

Different frequency ranges of a time varying signal, such as a LFP recorded on a single channel, can carry different information. However, it may be beneficial to average the spectral amplitudes over a larger frequency band whose sub-bands have homogeneous modulations in order to reduce the stochastic noise. Furthermore, some frequencies may not carry any information relevant to the studied behavior. To find LFP frequency bands that contain homogeneous modulations relevant to reach and grasp movements, we explored the modulation strength of all possible frequency bands, characterized by their bottom (minimum) and top (maximum) frequencies, in response to GO (method described in details below). For each dataset, three bands with homogenous modulations emerged: a low (1.5-4.5 Hz), an intermediate $(\sim 10-40 \mathrm{~Hz})$ and a high frequency band $(\sim 80-250 \mathrm{~Hz})$. For each monkey, these bands largely overlapped for different datasets. To preserve phase information that may be present in the low frequency field potentials (Ball et al., 2009; Hammer et al., 2013; Pistohl et al., 2012), instead of using the amplitude modulations of the identified low frequency bands, we used the low-pass filtered LFPs as a low frequency component of the recorded LFPs ( $L F C$ ). Identified intermediate and high frequency bands for each dataset were used to determine one intermediate and one high frequency band for each monkey. 
Amplitude modulations in these bands were used as intermediate and high frequency components of LFPs (IFC and HFC). LFC, IFC and HFC were then used as features in the classification and decoding analyses described below.

To explore the spectrotemporal LFP modulations, we first calculated spectral amplitudes $a\left(c h_{r e f}, n_{f}, t\right)$ in each common average referenced LFP channel $\mathrm{ch}_{\text {ref }}$ using $333 \mathrm{~ms}$ windows by applying a Hamming window and a Fourier transform (Milekovic et al., 2012, 2013). The window size of $333 \mathrm{~ms}$ was selected as a compromise between a spectral precision of $3 \mathrm{~Hz}$ and a temporal precision of $333 \mathrm{~ms}$. Windows overlapped by $313 \mathrm{~ms}$ to provide one amplitude estimate every $20 \mathrm{~ms}$. To ensure causality, time $t$ of the amplitude estimate was equal to the last temporal point of the window. The amplitudes $a\left(c h_{r e f}, n_{f}, t\right)$ in each frequency bin $n_{f}$ were then normalized by dividing them by the mean amplitude over the session in the corresponding frequency bin. This was done to account for the decrease in amplitudes with the increasing frequencies and to account for the changes in the LFP activity from one session to the next. Classification and decoding algorithms used in this study assumed normal distributions of the features. However, the distribution of normalized amplitudes on a single channel was skewed towards higher amplitudes. To make the distribution of the amplitudes closer to normal, we calculated the square root of the normalized amplitudes (SQNA) and used those in further analysis.

$\operatorname{SQNA}\left(\operatorname{ch}_{r e f}^{\prime}, n_{f}, t\right)=\sqrt{\frac{a\left(c h_{r e f}^{\prime}, n_{f}, t\right)}{\left\langle a\left(c h_{r e f}^{\prime}, n_{f}, t\right)\right\rangle_{\text {Session }}}}$

where \langle\rangle$_{\text {Session }}$ operator marks the mean over all time points in a given session. Examples of SQNA aligned on the GO and averaged over all trials from different trial types for an individual channel from the ref' subset can be seen in the left column of Fig. 3. We then calculated the band-mean SQNA (BMSQNA) for all possible continuous frequency bands. Each continuous frequency band was characterized by the bottom and top frequency $\left(f_{B}\right.$ and $f_{T}$ ), i.e. the bottom and top frequency bin $\left(n_{f B}\right.$ and $\left.n_{f T}\right)$ of the frequency band.

$\operatorname{BMSQNA}\left(\operatorname{ch}_{r e f}^{\prime}, f_{B}, f_{T}, t\right)=\frac{1}{n_{f_{T}}-n_{f_{B}}+1} \sum_{n_{f}=n_{f_{B}}}^{n_{f_{T}}} \operatorname{SQNA}\left(\operatorname{ch}_{r e f}^{\prime}, n_{f}, t\right)$.

If the amplitude modulations in response to an external stimulus are homogenous over a frequency band and the noise is stochastic, the strength of the modulation of that band will be higher than any frequency sub-band. To evaluate the strength of the modulation compared to baseline following $\mathrm{GO}$ for each continuous band, we calculated the signal-to-noise ratio (SNR; procedure described in Milekovic et al., 2012) between the GO triggered BMSQNA within the period from GO until $500 \mathrm{~ms}$ after GO and the BMSQNA collected over the whole dataset. This procedure was performed for each trial type in a given dataset separately.

$$
\begin{aligned}
& \mu_{\text {Type }}\left(c h_{r e f}^{\prime}, f_{B}, f_{T}, \tau\right)=\frac{1}{n_{E^{T y p e}}} \sum_{i=1}^{n_{E \text { Type }}} B M S Q N A\left(c h_{r e f}^{\prime}, f_{B}, f_{T}, t_{E_{i}^{T y p e}}+\tau\right) \\
& \sigma_{\text {Type }}\left(c h_{\text {ref }}^{\prime}, f_{B}, f_{T}, \tau\right) \\
& =\sqrt{\frac{1}{n_{E^{T} \text { Tpe }}-1} \sum_{i=1}^{n_{E_{\text {IJpe }}}}\left(B M S Q N A\left(c h_{\text {ref }}^{\prime}, f_{B}, f_{T}, t_{E_{i}^{\text {Type }}}+\tau\right)-\mu_{\text {Type }}\left(c h_{\text {ref }}^{\prime}, f_{B}, f_{T}, \tau\right)\right)^{2}} \\
& \mu_{\text {Mean }}\left(c h_{\text {ref }}^{\prime}, f_{B}, f_{T}\right)=\left\langle\operatorname{BMSQNA}\left(c h_{\text {ref }}^{\prime}, f_{B}, f_{T}, t\right)\right\rangle_{\text {Dataset }} \\
& \sigma_{\text {Mean }}\left(c h_{\text {ref }}^{\prime}, f_{B}, f_{T}\right)=\left\langle\left(B M S Q N A\left(c h_{\text {ref }}^{\prime}, f_{B}, f_{T}, t\right)-\mu_{\text {Mean }}\left(c h_{\text {ref }}^{\prime}, f_{B}, f_{T}\right)\right)^{2}\right\rangle_{\text {Dataset }} \\
& \operatorname{SNR}_{\text {Type }}\left(c h_{\text {ref }}^{\prime}, f_{B}, f_{T}, \tau\right)=\frac{\left|\mu_{\text {Type }}\left(c h_{\text {ref }}^{\prime}, f_{B}, f_{T}, \tau\right)-\mu_{\text {Mean }}\left(c h_{\text {ref }}^{\prime}, f_{B}, f_{T}\right)\right|}{\sigma_{\text {Type }}\left(c h_{\text {ref }}^{\prime}, f_{B}, f_{T}, \tau\right)+\sigma_{\text {Mean }}\left(c h_{\text {ref }}^{\prime}, f_{B}, f_{T}\right)} \quad 0 \leq \tau \leq 500 \mathrm{~ms}
\end{aligned}
$$

where $t_{E_{i}^{\text {Type }}}$ is the time of the $i$-th GO event for the trial type Type, $n_{E^{\text {Type }}}$ is the number of GO events for the trial type Type, $\tau$ is the time within the defined period and \langle\rangle$_{\text {Dataset }}$ operator calculates the mean over all time points in a given dataset. We then calculated the frequency band SNR by first taking the maximum over all time points in the defined period and then taking the mean over all channels in the common average set and all trial types present in the dataset.

$\operatorname{SNR}\left(f_{B}, f_{T}\right)=\frac{1}{N_{\text {ref }}^{\prime}} \frac{1}{n_{\text {Type }}} \sum_{\text {ch }=1}^{N_{\text {ref }}^{\prime}} \sum_{\text {Type }} \max _{\tau} \arg \left(\operatorname{SNR}_{\text {Type }}\left(\operatorname{ch}_{\text {ref }}^{\prime}, f_{B}, f_{T}, \tau\right)\right)$

where $n_{\text {Type }}$ is the number of trial types in the dataset. SNR was defined in this way in order to approximate the strength of reach-related modulation common over all trial types and dependent only on the frequency band. We identified three distinct local maxima of $\operatorname{SNR}\left(f_{B} f_{T}\right)$ corresponding to: (i) low frequencies $(1.5-4.5 \mathrm{~Hz}$ ), (ii) intermediate frequencies $(\sim 10-\sim 40 \mathrm{~Hz})$ and (iii) high frequencies $(\sim 80-250 \mathrm{~Hz})$ (Fig. 3, right column), indicating the existence of three distinct components of the LFP signals related to the complex reach-to-grasp movements, each present in a separate frequency band.

To represent the LFP signal components in the intermediate and high frequencies, we defined the intermediate frequency component (IFC) and the high frequency component (HFC) as the BMSQNA for the frequency band selected by taking the mean bottom and mean top frequencies of the intermediate and high frequency $\operatorname{SNR}\left(f_{B} f_{T}\right)$ local maxima over all datasets for each monkey separately (Table 3).

$$
\begin{aligned}
& { }_{\text {Monkey }}^{\mathrm{CMP}} n_{B}=\text { round }\left(\frac{1}{n_{\text {Dataset }}^{\text {Monkey }}} \sum_{i=1}^{\substack{\text { Monkey } \\
\text { Dataset }}} \mathrm{CMP}_{\text {Monkey }} n_{B_{i}}\right) \\
& { }_{\text {Monkey }}^{\mathrm{CMP}} n_{T}=\text { round }\left(\frac{1}{n_{\text {Dataset }}^{\text {Monky }}} \sum_{i=1}^{n_{\text {Dontaset }}^{\text {Monket }}} \mathrm{CMP} \text { Monkey } n_{T_{i}}\right) \\
& \operatorname{CMP}_{\text {Monkey }}\left(\operatorname{ch}_{\text {ref }}^{\prime}, t\right)=\operatorname{BMSQNA}\left(\operatorname{ch}_{\text {ref }}^{\prime}, f_{B}\left(\begin{array}{l}
\mathrm{CMP} \\
\text { Monkey }
\end{array} n_{B}\right), f_{T}\left(\begin{array}{l}
\text { Monkey } \\
\text { Monk }
\end{array}\right), t\right)
\end{aligned}
$$

where $C M P_{\text {Monkey }}$ is either IFC or HFC for monkey Monkey, ${ }_{\text {Monkey }}{ }_{B_{i}}$ and ${ }_{\text {Monkey }}^{\mathrm{CMP}} n_{T_{i}}$ are the bottom and top frequency bins defining the band that gave the local $\operatorname{SNR}\left(f_{B}, f_{T}\right)$ for component CMP and monkey Monkey for the $i$-th dataset, $n_{\text {Dataset }}^{\text {Monkey }}$ is the number of dataset for the monkey Monkey, and round() is the operator that gives the closest integer.

To preserve the information encoded in the phase of the low frequency LFPs, we defined the low frequency component ( $L F C$ ) as a common averaged referenced signal filtered using a 2nd order SavitzkyGolay filter (Savitzky and Golay, 1964; Steinier et al., 1972) with a time window of $335 \mathrm{~ms}$ and a delay of $30 \mathrm{~ms}$. To correct for the changes in the $L F C$ activity from one session to the next, we subtracted the mean LFC over each session.

$\operatorname{LFC}\left(\operatorname{ch}_{\text {ref }}^{\prime}, t\right)=\operatorname{sgolay}\left(\bar{\Phi}\left(\operatorname{ch}_{\text {ref }}^{\prime}, t\right)\right)-\left\langle\operatorname{sgolay}\left(\bar{\Phi}\left(\operatorname{ch}_{\text {ref }}^{\prime}, t\right)\right)\right\rangle_{\text {Session }}$

where sgolay is the Savitzky-Golay filter operator. Examples of the three LFP components are provided in Fig. 4.

\section{Classification of neural responses}

We estimated the amount of information related to the 2 grips (SG and PG) and the 2 load forces (LF and HF) contained in the LFP signals for different time points before and after (i) the CUE, (ii) the GO and (iii) object movement onset by performing classification of individual trials with respect to the grip type or load force based on LFPs recorded 

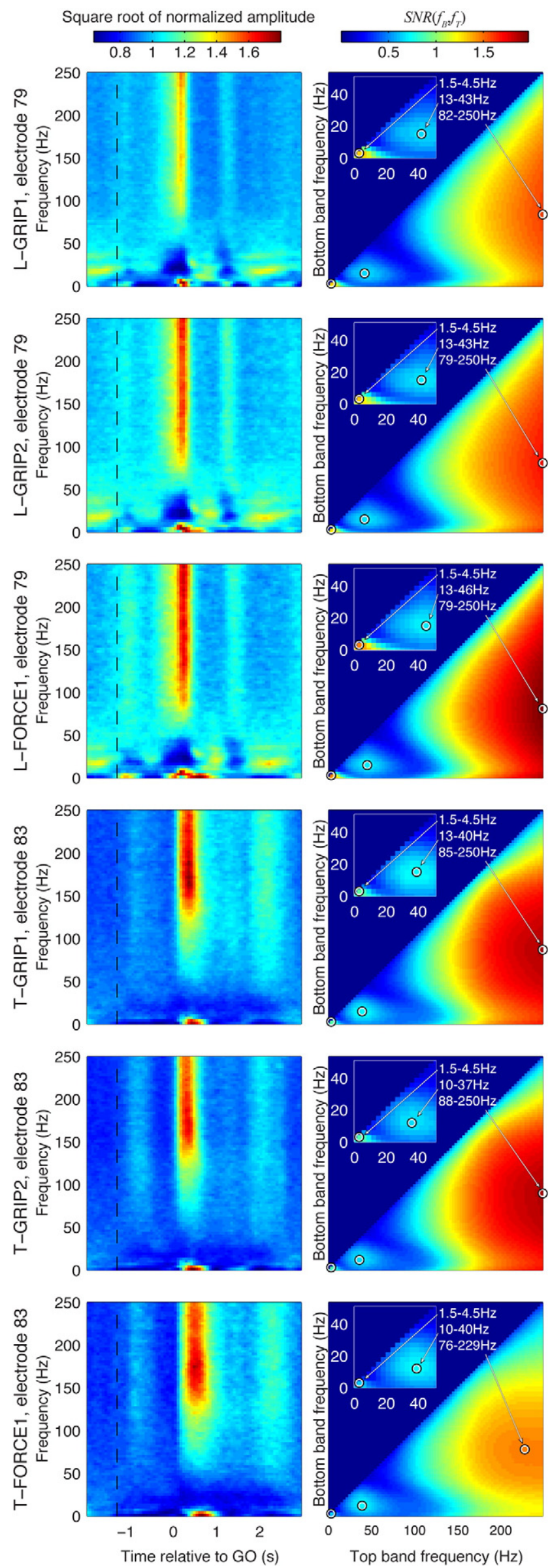

Table 3

Frequency bins and frequencies of the SNR local maxima for each dataset and averaged over datasets.

\begin{tabular}{|c|c|c|c|c|}
\hline \multirow[t]{2}{*}{ Dataset } & \multicolumn{2}{|l|}{ IFC } & \multicolumn{2}{|l|}{ HFC } \\
\hline & $\overline{b S}$ (bSfreq) & bE (bEfreq) & $\overline{b S}$ (bSfreq) & bE (bEfreq) \\
\hline L-GRIP1 & $6(13 \mathrm{~Hz})$ & $15(43 \mathrm{~Hz})$ & $29(82 \mathrm{~Hz})$ & $84(250 \mathrm{~Hz})$ \\
\hline L-GRIP2 & $6(13 \mathrm{~Hz})$ & $15(43 \mathrm{~Hz})$ & $28(79 \mathrm{~Hz})$ & $84(250 \mathrm{~Hz})$ \\
\hline L-FORCE1 & $6(13 \mathrm{~Hz})$ & $16(46 \mathrm{~Hz})$ & $28(79 \mathrm{~Hz})$ & $84(250 \mathrm{~Hz})$ \\
\hline Average & $6(13 \mathrm{~Hz})$ & $15(43 \mathrm{~Hz})$ & $28(79 \mathrm{~Hz})$ & $84(250 \mathrm{~Hz})$ \\
\hline T-GRIP1 & $6(13 \mathrm{~Hz})$ & $14(40 \mathrm{~Hz})$ & $30(85 \mathrm{~Hz})$ & $84(250 \mathrm{~Hz})$ \\
\hline T-GRIP2 & $5(10 \mathrm{~Hz})$ & $13(37 \mathrm{~Hz})$ & $31(88 \mathrm{~Hz})$ & $84(250 \mathrm{~Hz})$ \\
\hline T-FORCE1 & $5(10 \mathrm{~Hz})$ & $14(40 \mathrm{~Hz})$ & $27(76 \mathrm{~Hz})$ & $77(229 \mathrm{~Hz})$ \\
\hline Average & $5(10 \mathrm{~Hz})$ & $14(40 \mathrm{~Hz})$ & $29(82 \mathrm{~Hz})$ & $82(244 \mathrm{~Hz})$ \\
\hline
\end{tabular}

throughout the trial. We first z-scored all normalized LFP components over the whole dataset:

$\operatorname{CMP}^{*}\left(\operatorname{ch}_{r e f}^{\prime}, t\right)=\frac{\operatorname{CMP}\left(\operatorname{ch}_{r e f}^{\prime}, t\right)-\left\langle\operatorname{CMP}\left(\operatorname{ch}_{r e f}^{\prime}, t\right)\right\rangle_{\text {Dataset }}}{\operatorname{std}\left(\operatorname{CMP}\left(\operatorname{ch}_{\text {ref }}^{\prime}, t\right)\right)_{\text {Dataset }}}$

where $\operatorname{std}()_{\text {Dataset }}$ is the standard deviation operator acting over all time points in a given dataset. For a given temporal offset $t_{D}$, we generated a labeled set of trials $\operatorname{tr}_{i}$ composed of $L F C$, IFC or HFC at time $t_{D}$ with respect to the time of each event.

$\operatorname{tr}_{i}\left(t_{D}, C M P\right)=\left[\begin{array}{c}C M P^{*}\left(c h_{r e f}^{\prime}(1), t_{i}+t_{D}\right) \\ \vdots \\ C M P^{*}\left(c h_{r e f}^{\prime}\left(N_{r e f}^{\prime}\right), t_{i}+t_{D}\right)\end{array}\right]$

where CMP is one of $L F C$, IFC or HFC; and $t_{i}$ is the time of the event (either CUE or object movement onset) in the $i$-th trial.

The classification was performed using a regularized linear discriminant analysis (RLDA; Friedman, 1989) that classified test trials according to the distributions of the labeled trials used to calibrate its model. To evaluate the classification accuracy (CA), we used a 50 times repeated five-fold estimation with five time repeated four-fold crossvalidation for selection of the regularization parameter, described in the following. We first shuffled the order of the trials in the dataset and then divided the dataset into five equally sized parts. Four parts were used to select the regularization parameter out of $0,0.001,0.1$, $0.3,0.5,0.7,0.9$ and 0.99 using four-fold cross-validation repeated five times, each time randomizing the order of the trials. Crossvalidation was repeated to reduce the influence of the bias that can arise by having a subset of trials for which one value of the parameter is suitable, but does not generalize over the whole dataset. We then calibrated the model on these four parts and used the remaining part to evaluate the CA. This procedure was repeated five times, each time selecting a different part for CA evaluation. The whole process was repeated 50 times, each time randomly shuffling all trials. These repetitions were performed to reduce the influence of the bias that can arise from choosing a subset of trials particularly suitable to the calibrated model that does not generalize for other trials. In total, this procedure produced 250 different CA estimates. The final CA estimate was gained by calculating the mean of these 250 values. To investigate the temporal evolution of the

Fig. 3. Spectral analysis of LFP responses for different datasets (one in each row). Left panels show mean spectrograms of the LFP responses recorded on one electrode, averaged over all trials of a dataset aligned to GO. Color coded is the squared root of the amplitude normalized to the mean amplitude of each frequency bin over the whole dataset. Black broken line shows the onset of the informative cue (CUE). Right panels show the frequency band signal-to-noise ratio $\left(\operatorname{SNR}\left(f_{B} f_{T}\right)\right)$ for each dataset. Insets in the top left corner show the same $S N R\left(f_{B} f_{T}\right)$ zoomed in to the lower frequencies. Black circles and arrows show three identified local maxima of $\operatorname{SNR}\left(f_{B}, f_{T}\right)$. Maxima containing intermediate and high frequency bands were used to define the frequency band of the intermediate and high frequency components (IFC and HFC, respectively). Top and bottom frequencies of the frequency bands for which $\operatorname{SNR}\left(f_{B} f_{T}\right)$ has a local maximum $\left(f_{B}\right.$ and $f_{T}$ respectively) are written in white. 


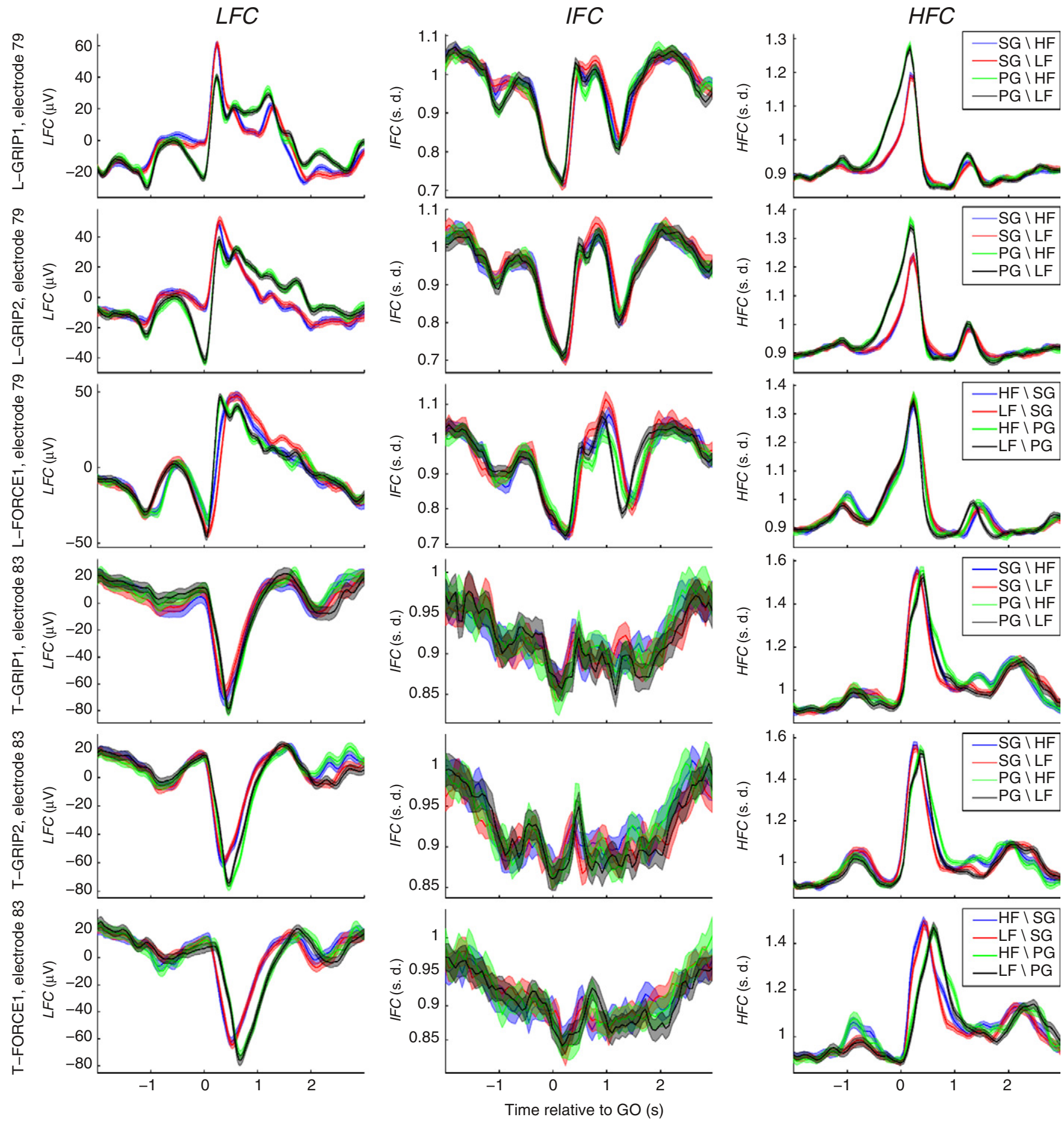

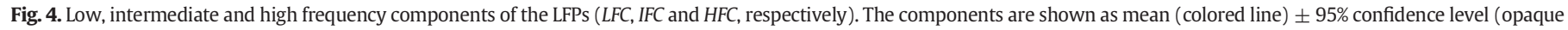

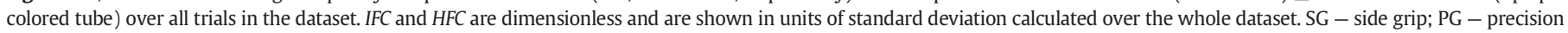
grip; LF - low force; HF - high force.

grip and force related information in the LFPs, CA was calculated for $t_{D}$ from $500 \mathrm{~ms}$ before the CUE to $1000 \mathrm{~ms}$ after the GO and from $500 \mathrm{~ms}$ before the object movement onset to $1000 \mathrm{~ms}$ after the object movement onset in steps of $10 \mathrm{~ms}$.

We chose to use RLDA due to its simplicity, which allowed for rapid model calibration and testing. In addition, RLDA can we be used to calibrate accurate classification models even when covariances of the features cannot be accurately determined due to sparse sampling of the feature space (Hastie et al., 2009). In our study, CA was evaluated for a large number of time points and conditions. Furthermore, a large number of features were used for classification, leading to sparse sampling of the feature space. Therefore, using more complex methods may have resulted in lower CA estimates and substantially longer computation times (for an example, see Milekovic et al., 2013).

To evaluate the statistical significance of obtained CA values, we used Barnard's test (Barnard, 1945) to calculate the p-value. Barnard's test is used to test the significance of $2 \times 2$ tables, such as confusion matrices obtained as a result of classification between two classes. It is a 
non-parametric alternative to the Fisher's exact test (Fisher, 1922). The calculation was performed for each combination of (i) a dataset, (ii) an event (the CUE, the GO or the object movement onset), (iii) a component, (iv) a classification task (SG vs. PG or HF vs. LF) and (v) a temporal offset; which resulted with 13,176 statistical tests for each monkey. To correct for multiple testing and to control the number of falsely rejected null hypotheses, we used the Benjamini-Hochberg procedure (Benjamini and Hochberg, 1995) with a correction for dependent statistics (Benjamini and Yekutieli, 2001) to set the false discovery rate for one monkey at the level of $1 \%$ for all classification statistical tests.

To identify the $t_{D}$ for which a LFP component gave a statistically significant CA with higher certainty, we required that the CA is statistically significant for that $t_{D}$ and the next consecutive $t_{D}$, i.e. for two consecutive CA measurements that were 10 ms apart.

\section{Continuous temporal offset-resolved decoding of grip parameters}

Our intention was to estimate the extent to which the hand position and the finger pressure forces are encoded in the LFPs recorded in macaque primary motor (MI) and dorsal premotor (PMd) cortices. Therefore, we first calibrated a linear transformation between grip parameters and LFP components (referred to as model) on one part of the dataset and then used the model to decode grip parameters from LFP components in the remainder of the dataset. Decoding was performed only during the active phase of the trials since this was the only period when the grip parameters could be determined.

Decoding was performed using linear ridge regression (Hastie et al., 2009) with a temporal offset between the LFP signals and the grip parameter $(\Delta t)$ ranging from $-2.3 \mathrm{~s}$ to $2.3 \mathrm{~s}$ in steps of $0.1 \mathrm{~s}$. Model calibration can be described as follows. We first z-scored all LFP components (Eq. (11)) and all grip parameters GP over the whole dataset.

$G P(t)^{*}=\frac{G P(t)-\langle G P(t)\rangle_{\text {Dataset }}}{s t d(G P(t))_{\text {Dataset }}}$.

Let $b_{1}, \ldots, b_{N_{r e f}^{\prime}}$ be the coefficients of our model. Then the relation between the grip parameter at time $t$ and the LFPs is as follows:

$G P(t)^{*}=\sum_{j=1}^{N_{r e f}^{\prime}} b_{j} C M P^{*}\left(c h_{r e f}^{\prime}(j), t+\Delta t\right)$

$\forall t,\left.i\right|_{A C} ^{\mathrm{GP}} t_{i}^{B} \leq t<<_{A C}^{\mathrm{GP}} t_{i}^{E}$

where ${ }_{A C}^{G P} t_{i}^{B}$ and ${ }_{A C}^{G P} t_{i}^{E}$ are the beginning and the end of the active phase for the grip parameter GP during the $i$-th trial. The coefficients were calculated using the relation:

$X=\left[\begin{array}{ccc}C M P^{*}\left(c h_{r e f}^{\prime}(1), t_{1}+\Delta t\right) & \ldots & C M P^{*}\left(c h_{r e f}^{\prime}\left(N_{r e f}^{\prime}\right), t_{1}+\Delta t\right) \\ \vdots & & \\ C M P^{*}\left(c h_{r e f}^{\prime}(1), t_{M}+\Delta t\right) & \ldots & C M P^{*}\left(c h_{r e f}^{\prime}\left(N_{r e f}^{\prime}\right), t_{M}+\Delta t\right)\end{array}\right]$, $Y=\left[\begin{array}{c}G P\left(t_{1}\right)^{*} \\ \vdots \\ G P\left(t_{M}\right)^{*}\end{array}\right], B=\left[\begin{array}{c}b_{1} \\ \vdots \\ b_{N_{r e f}^{\prime}}^{\prime}\end{array}\right]$

$B=Y^{T} \cdot X \cdot \operatorname{inv}\left(X^{T} \cdot X+\gamma \cdot \operatorname{mean}\left(\operatorname{diag}\left(X^{T} \cdot X\right)\right) \cdot I\right)$

where $t_{1}, \ldots, t_{M}$ are the all times satisfying the condition from Eq. (15), $\gamma$ is the regularization parameter, mean is the mean operator, diag is the diagonalization operator and $I$ is the $N \times N$ identity matrix.
Decoding accuracy was evaluated using the fraction of accounted variance (FAV; Fagg et al., 2009):

$F A V=1-\frac{V\left(G P-G P_{D E C}\right)}{V(G P)}$

where $G P_{D E C}$ is the decoded grip parameter and the $V()$ is the variance operator calculated over all time points. We calculated FAV by a threefold estimation with three-fold cross-validation to select the regularization parameter $\gamma$ from the following set of values: 0, 0.032, 0.049, 0.077, $0.119,0.186,0.289$ (see section Classification of neural responses for detailed description of the procedure). For each dataset, this procedure gave three estimates of the $F A V$ and the reported $F A V$ is their mean. In the remainder of the text, we refer to these $F A V$ values as "execution FAV".

We decoded grip parameters during the dynamic phase exclusively from the LFPs during the preparatory period. Decoding was performed using the following relation between a grip parameter GP and a LFP component CMP:

$G P\left(\underset{D Y N}{G P} t_{i}^{B}+t\right)^{*}=\sum_{j=1}^{N_{r e f}^{\prime}} b_{j} C M P^{*}\left(c h_{r e f}^{\prime}(1), t_{i}^{C U E}+\tau+t\right)$

$\forall t, i \mid 0 \leq t \leq \leq_{D Y N}^{\mathrm{GP}} t_{i}^{E}-{ }_{D Y N}^{\mathrm{GP}} t_{i}^{B}, 0 \leq \tau+t<t_{i}^{G O}-t_{i}^{C U E}$

where $\tau$ is the temporal offset between the CUE and start of the period during which LFPs were used to decode the grip parameter in the dynamic phase; and ${ }_{D Y N}^{G P} t_{i}^{B}$ and ${ }_{D Y N}^{G P} t_{i}^{E}$ are the beginning and the end of the dynamic phase for grip parameter GP in $i$-th trial, respectively. Note that $\tau$, unlike $\Delta t$, is trial dependent when considering temporal difference between the grip parameter and LFPs used to decode it. This approach tests whether the grip parameter during the dynamic phase of the trial is encoded in the LFPs during the delay period with the similar rate of change as the grip parameter, taking into account a delay $\tau$ with respect to CUE. Used $\tau$ values were between $0 \mathrm{~ms}$ and $1200 \mathrm{~ms}$ in steps of $30 \mathrm{~ms}$. Models were built using the linear ridge regression algorithm (Eq. (17)) using the following matrix identities:

$$
\begin{aligned}
& X=\left[\begin{array}{ccc}
C M P^{*}\left(c h_{r e f}^{\prime}(1), t_{1}+\tau\right) & \ldots & C M P^{*}\left(c h_{r e f}^{\prime}\left(N_{r e f}^{\prime}\right), t_{1}+\tau\right) \\
\vdots & \vdots & \\
C M P^{*}\left(c h_{r e f}^{\prime}(1), t_{M}+\tau\right) & \ldots & C M P^{*}\left(c h_{r e f}^{\prime}\left(N_{r e f}^{\prime}\right), t_{M}+\tau\right)
\end{array}\right], \\
& Y=\left[G P\left({ }_{D Y N} t_{i}^{B}-t_{i}^{C U E}+t_{1}\right)^{*}: G P\left({ }_{D Y N} t_{i}^{B}-t_{i}^{\mathrm{CUE}}+t_{M}\right)^{*}\right], B=\left[\begin{array}{c}
b_{1} \\
\vdots \\
b_{N_{r e f}^{\prime}}
\end{array}\right]
\end{aligned}
$$

with $t_{1}, \ldots, t_{M}$ being all times $t$ satisfying conditions from Eq. (20). Decoding accuracy was evaluated using $F A V$, calculated by a five-fold estimation with four-fold cross-validation to select the regularization parameter $\gamma$ from the following set of values: 0, 0.032, 0.049, 0.077, 0.119, 0.186 , and 0.289 (see section Classification of neural responses for detailed description of the procedure). For each dataset, this procedure gave five estimates of the FAV and the reported FAV is their mean. In the remainder of the text, we refer to these $F A V$ values as "planning FAV".

To evaluate the FAV values for no relationship between the grip parameter and the LFPs (chance), we generated "randomly translated" grip parameter values by translating the grip parameter values during the active or dynamic phase by a random temporal shift in relation to the LFPs. These temporal translations were done in a cyclical manner: end of the grip parameter recordings was translated to the beginning of the recordings. From the visual inspection of the broadness of the central peak of the autocorrelation of the grip parameters, the minimum time of translation was set to be $10 \mathrm{~s}$ and $0.2 \mathrm{~s}$ for decoding active phase and dynamic phase, respectively. This procedure was done for each grip 
parameter, each $\Delta t$ or $\tau$ and each LFP component used for decoding independently. After the random translation, we calculated $F A V$ using the same procedure as for decoding the grip parameter. We repeated the procedure (randomly translating grip parameters and calculating decoding accuracy) 2000 and 5000 times for execution and planning $F A V$, thus providing a set of 2000 or 5000 random translation $F A V$ values. We calculated the p-value using the Monte Carlo method:

$p=\frac{N_{G E}+1}{N_{A L L}+1}$

where $N_{G E}$ is the number of random translation $F A V$ values greater or equal to the $F A V$ value obtained from decoding the grip parameter and $N_{A L L}=2000$ or 5000 is the total number of random translation FAV values. To correct for multiple testing (1692 and 1476 tests in total for decoding active and dynamic phase for each monkey, respectively) and to control the number of falsely rejected null hypotheses, we again used the Benjamini-Hochberg procedure with a correction for dependent statistics to set the false discovery rate for one monkey to the level of $1 \%$ for all active and dynamic decoding accuracy statistical tests separately.

\section{Selection of regularization parameters}

Our approach for selecting the regularization parameters can lead to over-fitting caused by biased selection of the regularization parameters. This can lead to lower CA and FAV estimates. A different approach (Fagg et al., 2009) separates a part of data to be used for validation of models with different values of the parameters. While this approach leads to an unbiased selection of parameters, it also reduces the part of the data used to build and test the model, which increases the variance of the selected parameters and, in turn, can lead to lower CA and FAV estimates as well. The tradeoff between the bias and the variance is dataset specific and cannot be estimated. Therefore, both biased and unbiased parameter selection will lead to reduced CA and FAV estimates, without a way to estimate which approach leads to a lower error.

We used between 29 and 83 simultaneously recorded channels of data to classify or decode parameters of grasping. Number of trials varied between 428 and 933, which is still substantially lower than the recommended power of 10 for each feature dimension, $\geq 10^{29}$ (Hastie et al., 2009). Even though our classification and decoding models both implemented regularization to account for this issue, we still opted to maximize the number of used trials in an effort to reduce the variance of the selected parameter values, rather than focusing on removing the bias.

\section{Results}

In this study, we identified three components of the LFP signals recorded from motor cortical areas of behaving macaque monkeys (low, intermediate and high frequency component; LFC, IFC and HFC) and used them to classify different grip types and load forces and to decode four different grip kinetic and kinematic parameters: thumb, index finger and middle finger pressure forces and, indirectly, hand position. These decoding analyses were conducted in two different tasks (Fig. 1): a grip cue task (GRIP) and a force cue task (FORCE). Given the anatomical landmarks visible during the implantation surgery, we were certain that arrays cover parts of the areas MI and PMd located on the surface of the precentral gyrus. However, due to the lack of a clear anatomical border between these two areas, we did not distinguish recordings originating from MI as opposed to those originating from PMd. Therefore, in the remainder of the text, we refer to the area covered by the array as "motor cortex".

\section{Behavioral results}

We analyzed three datasets for each monkey, two GRIP datasets (LGRIP1 and L-GRIP2 for monkey L; T-GRIP1 and T-GRIP2 for monkey $\mathrm{T}$ ) and one FORCE dataset (L-FORCE1 for monkey L; T-FORCE1 for monkey T). Each dataset contained several sessions recorded within three consecutive days and contained 487 or more correct trials (Table 2). From these, we excluded $1 \%$ of the trials for monkey $\mathrm{L}$ and $8 \%$ trials for monkey $\mathrm{T}$ due to RT + MT being above $1 \mathrm{~s}$ (see section Data processing for details about the exclusion procedure; Table 2). The mean duration ( $\pm 95 \%$ confidence interval of the mean) of RT + MT was $400 \pm 5 \mathrm{~ms}$ for monkey L and $686 \pm 10 \mathrm{~ms}$ for monkey T (monkey L: L-GRIP1: $332 \pm 3$ ms; L-GRIP2: $395 \pm 5$ ms; L-FORCE1: $490 \pm 8$ ms; monkey T: T-GRIP1: $614 \pm 8$ ms; T-GRIP2: $553 \pm 7$ ms; T-FORCE1: $746 \pm 10 \mathrm{~ms})$.

Finger pressure forces depended more strongly on the grip type than on the load force, as seen from the values of the linear fit coefficients $a_{G}$ and $a_{F}$ (Table 4). The dependence of the thumb and index pressure force on the grip type was 2.6 and 5.8 times higher than on the load force when averaged over all datasets, respectively. In the L-GRIP and LFORCE datasets, the monkeys pressed on the object with similar middle finger pressure forces on average in all four trial types, but still executed the pressure with large trial-to-trial variability. Thus, the linear model used to fit the pressure forces was not accurate in predicting them, as can be seen from low $r^{2}$ in Table 4. For monkey T, the middle finger pressure force was 22.5 times more dependent on the grip type than on the load force when averaged over all datasets. On the other hand, dependence of displacement on the loading force was 2.1 times higher than the dependence on the grip type when averaged over all datasets. This dependence is intuitive, since the applied finger pressure forces acted perpendicular to the direction of object movement and, therefore, had no effect on it. Load force was acting in the direction of object movement and, therefore, monkeys found it easier to move the object further if the load force was lower. Even so, the variability of the displacement could only weakly be explained by its dependence on the grip type and load force, as seen from the lower $r^{2}$ values as compared to $r^{2}$ values for thumb and index finger pressure forces.

\section{Classification of grip types and load forces from neural responses}

In the first step of our analysis, we estimated the amount of information in selected LFP components at different moments during the trial by classifying trials with respect to the two parameters of the task, the grip types and the load forces. LFPs used for classification were either aligned to CUE (and thus to GO since the period between them was constant) or to object movement onset (Figs. 5 and 6). The classification accuracy $(\mathrm{CA})$ became significant at different time instances during the trial. The times of the first significant CAs for the grip type (Grip-CA) and for the load force (Force-CA), and the times and CAs of the Grip-CA and Force-CA maxima are given in Tables 5 and 6. Below, we summarize the classification results. Since the temporal evolution of CA values for the low and high frequency components was similar, we grouped the description of their results.

\section{Grip cue task (GRIP)}

Low and high frequency components (LFC and HFC). As illustrated in Fig. 5 (top 4 panels; light red and green lines), for monkey L using LFPs aligned to CUE, Grip-CA first reached statistical significance within $110 \mathrm{~ms}$ after CUE and then rose quickly to reach a local maximum followed by a trough. For monkey T, Grip-CA reached significance after CUE later than for monkey L. Furthermore, during the delay period (epoch between CUE and GO) Grip-CA values were substantially lower for monkey $\mathrm{T}$ than for monkey $\mathrm{L}$ and stayed close to chance level (T-GRIP1) or increased only slightly throughout the delay period (T-GRIP2). 
Table 4

Coefficient values and $r^{2}$ values of fitting a linear of grip type and load force dependence of grip parameters.

\begin{tabular}{|c|c|c|c|c|c|c|c|c|c|c|c|c|}
\hline & \multicolumn{3}{|c|}{ Thumb } & \multicolumn{3}{|c|}{ Index finger } & \multicolumn{3}{|c|}{ Middle finger } & \multicolumn{3}{|c|}{ Displacement } \\
\hline & $a_{G}$ & $a_{F}$ & $r^{2}$ & $a_{G}$ & $a_{F}$ & $r^{2}$ & $a_{G}$ & $a_{F}$ & $r^{2}$ & $a_{G}$ & $a_{F}$ & $r^{2}$ \\
\hline L-GRIP & 1.54 & 0.80 & 0.65 & 5.01 & 1.05 & 0.95 & 0.14 & 0.26 & 0.07 & -1.39 & -2.33 & 0.17 \\
\hline L-FORCE & 1.74 & 0.52 & 0.78 & 4.78 & 0.59 & 0.94 & 0.21 & 0.28 & 0.07 & -2.03 & -3.51 & 0.41 \\
\hline T-GRIP & 2.80 & 1.38 & 0.83 & 5.59 & 1.24 & 0.96 & -1.50 & 0.14 & 0.74 & -0.10 & -0.39 & 0.01 \\
\hline T-FORCE & 1.96 & 0.63 & 0.73 & 5.12 & 0.90 & 0.96 & -1.42 & 0.04 & 0.79 & -1.18 & -2.36 & 0.21 \\
\hline
\end{tabular}

For both monkeys, Grip-CA rose before GO and reached a local maximum between $130 \mathrm{~ms}$ and $500 \mathrm{~ms}$ after GO. These results imply that low and high frequency components diverged for different grip types in expectation of the GO, rather than being a response to it. For monkey L, Grip-CA reached values of 1 or close to it, while the maximum Grip-CA for monkey T was slightly lower, between 0.81 and 0.92 . When LFPs
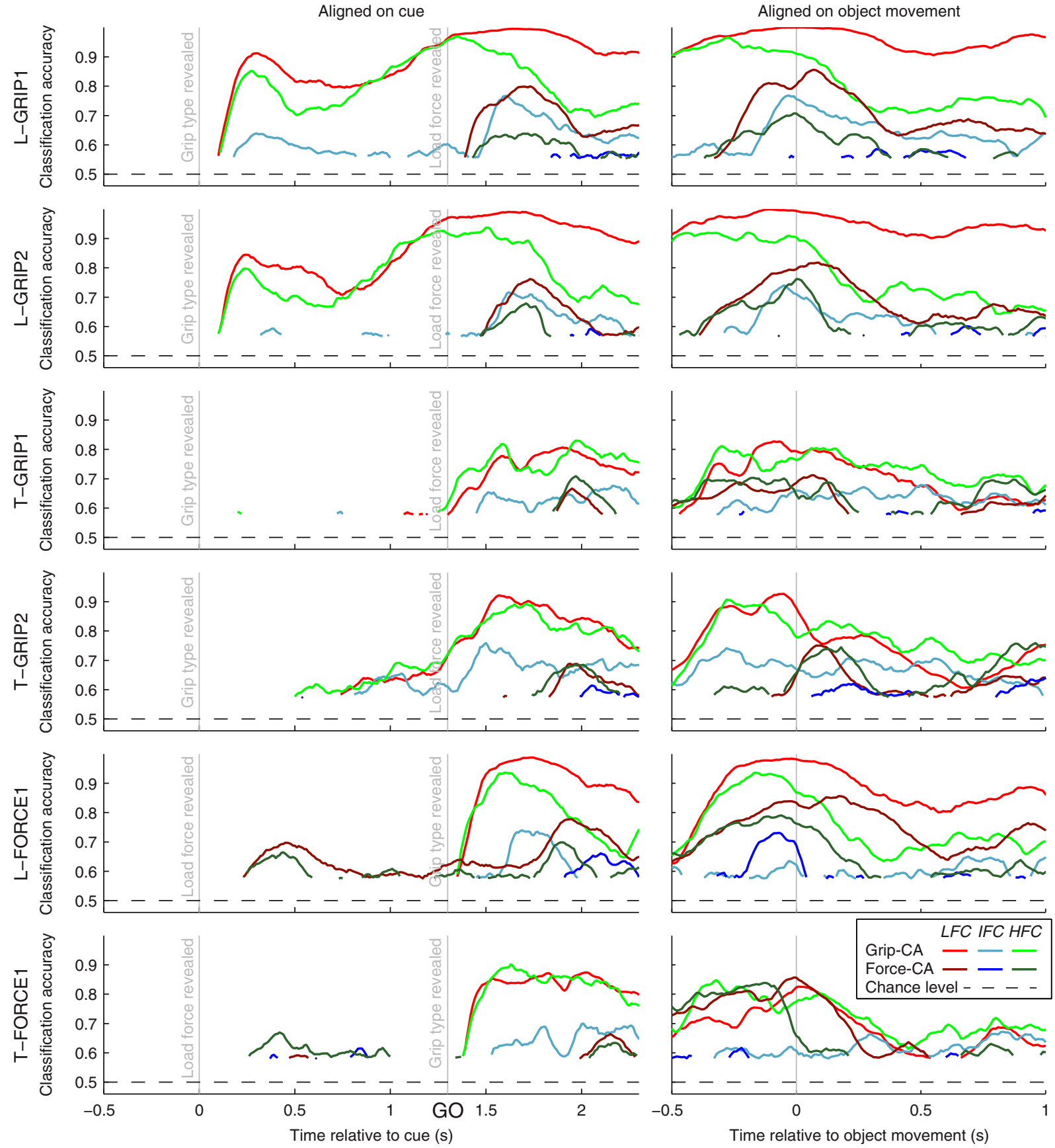

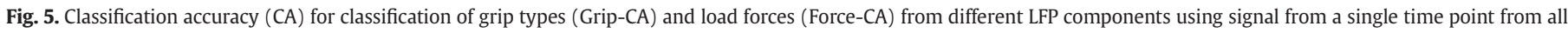

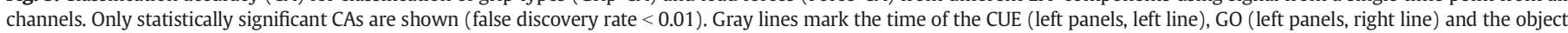
movement onset (right panels). Text on the gray lines shows the type of information revealed to the monkey at that moment. 

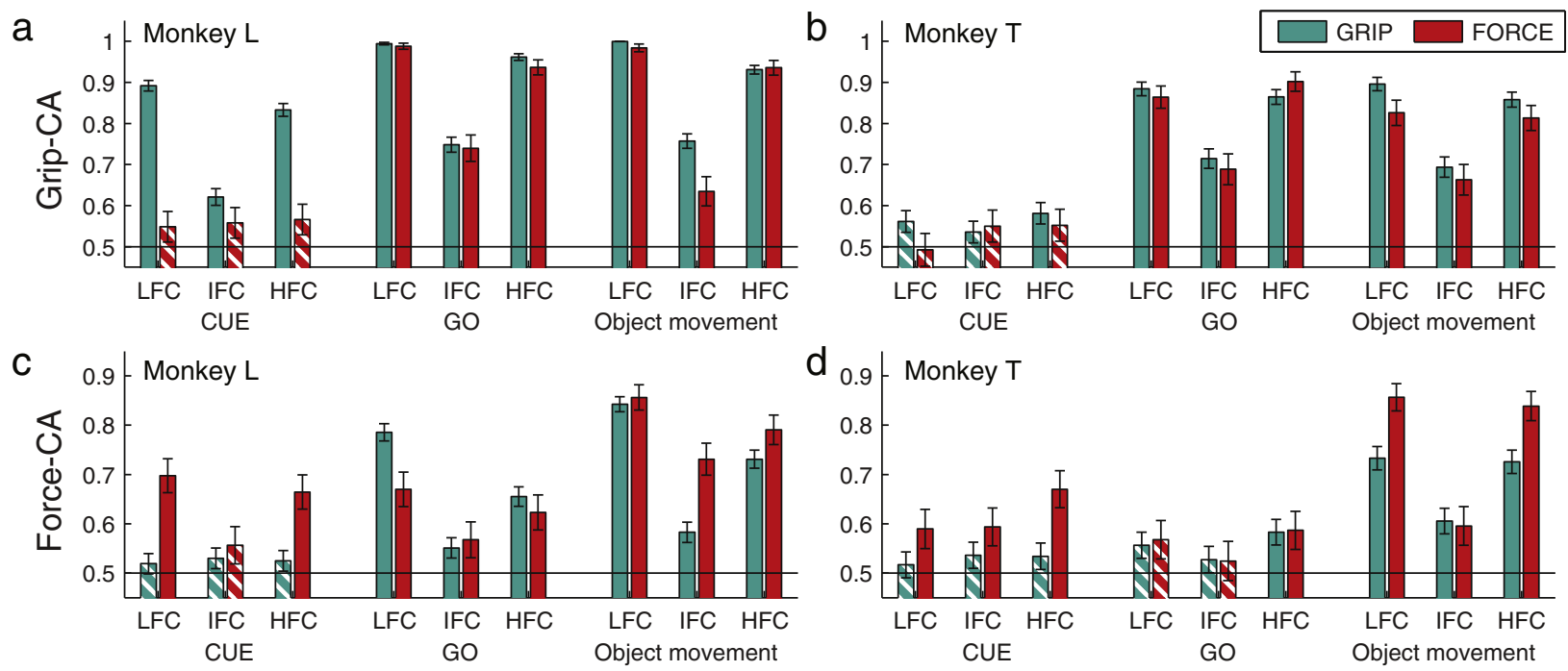

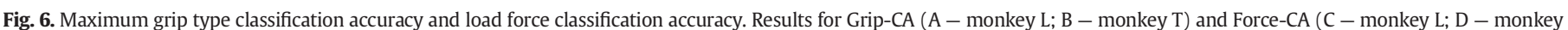

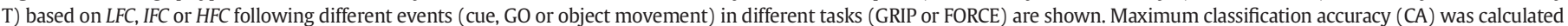
within the temporal epoch from $200 \mathrm{~ms}$ before the event and until $500 \mathrm{~ms}$ after the event. Bars marked with white diagonal lines show insignificant CAs.

were aligned to object movement onset, a Grip-CA maximum was reached at or slightly before it, with maximum values of above 0.92 for monkey L and between 0.81 and 0.93 for monkey $\mathrm{T}$.

For LFPs aligned to CUE, Force-CA (Fig. 5, top 4 panels; dark red and green lines) did not reach significance before GO for both monkeys. This was consistent with the task design, since monkeys did not have any information about the load force before GO. First significant Force-CAs were obtained within $180 \mathrm{~ms}$ and $570 \mathrm{~ms}$ after GO for monkey L and monkey T, respectively. Force-CAs then quickly increased and reached a local maximum, with Force-CAs substantially higher for monkey $\mathrm{L}$ than for monkey T. When LFPs were aligned to object movement onset, Force-CA maxima occurred close to the object movement onset and were significantly higher than the Force-CA maxima obtained from LFPs aligned to CUE ( $\mathrm{p}<0.01$, Wilcoxon signed rank test).

Intermediate frequency component (IFC). For both monkeys, Grip-CA and Force-CA from the intermediate frequency component (Fig. 5, top 4 panels; light and dark blue lines) were lower and became significant later than Grip-CA and Force-CA from low and high frequency components. For LFPs aligned to CUE, Grip-CA from intermediate frequency component rose fast after $\mathrm{GO}$ and had narrow peaks, unlike Grip-CA from low and high frequency components that had wide peaks after GO. However, times of Grip-CA maxima from all three components were similar. Force-CA from the intermediate frequency component in all GRIP datasets barely reached significance and never crossed 0.62 .

\section{Force cue task (FORCE)}

Low and high frequency components (LFC and HFC). As shown in the lower two panels of Fig. 5, for LFPs aligned to CUE, Force-CA became significant within $260 \mathrm{~ms}$ after the CUE, rose quickly and reached a local maximum, with the exception for Force-CA from low frequency component in T-FORCE1 dataset that became significant only $470 \mathrm{~ms}$ after the CUE. For monkey L, Force-CA maxima during the delay period were lower than the Grip-CA maxima in GRIP datasets. After the maximum, Force-CA slowly dropped and then rose again to reveal a peak at between $600 \mathrm{~ms}$ and $870 \mathrm{~ms}$ after GO, similar to Grip-CA for monkey L in GRIP datasets. When aligned to object movement, Force-CA increased substantially for both monkeys when compared to Force-CA from signals aligned to CUE.

Grip-CAs from LFPs aligned to CUE were not significant before GO, as consistent with the task design in FORCE datasets. After GO, Grip-CAs became significant as early as $50 \mathrm{~ms}$ after GO for monkey L and $80 \mathrm{~ms}$ for monkey T. In our experiments, monkeys were trained to expect the GO signal exactly $1 \mathrm{~s}$ after the CUE signal disappeared and knew where the GO cue would appear. Our experimental design may have prepared the neural pathways for the upcoming information and, thus, expedited the transmission of the information. This result is in agreement with a study from Santhanam et al. (2006) who found significant decoding of movement-related information from PMd neural activity as early as 50-70 ms after presentation of the visual

Table 5

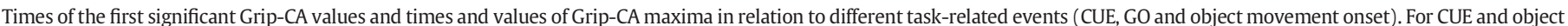

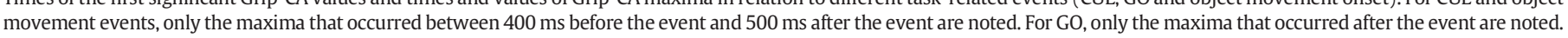

\begin{tabular}{|c|c|c|c|c|c|c|c|c|c|c|c|c|c|c|}
\hline \multirow[t]{3}{*}{ Dataset } & \multicolumn{6}{|l|}{ CUE } & \multicolumn{4}{|l|}{ GO } & \multicolumn{4}{|c|}{ Object movement onset } \\
\hline & \multicolumn{2}{|c|}{$\begin{array}{l}\text { Time of first } \\
\text { significant } \\
\text { Grip-CA (ms) }\end{array}$} & \multicolumn{2}{|c|}{$\begin{array}{l}\text { Time of } \\
\text { Grip-CA } \\
\text { maximum } \\
(\mathrm{ms})\end{array}$} & \multicolumn{2}{|c|}{$\begin{array}{l}\text { Maximum } \\
\text { Grip-CA value }\end{array}$} & \multicolumn{2}{|c|}{$\begin{array}{l}\text { Time of } \\
\text { Grip-CA } \\
\text { maximum } \\
(\mathrm{ms})\end{array}$} & \multicolumn{2}{|c|}{$\begin{array}{l}\text { Maximum } \\
\text { Grip-CA value }\end{array}$} & \multicolumn{2}{|c|}{$\begin{array}{l}\text { Time of Grip-CA } \\
\text { maximum (ms) }\end{array}$} & \multicolumn{2}{|c|}{$\begin{array}{l}\text { Maximum } \\
\text { Grip-CA value }\end{array}$} \\
\hline & LFC & HFC & LFC & HFC & LFC & HFC & LFC & HFC & LFC & HFC & LFC & HFC & LFC & HFC \\
\hline L-GRIP1 & 100 & 110 & 300 & 280 & 0.91 & 0.85 & 360 & 50 & 1 & 0.97 & -30 & -190 & 1 & 0.94 \\
\hline L-GRIP2 & 110 & 100 & 240 & 240 & 0.84 & 0.80 & 390 & 210 & 0.99 & 0.94 & -110 & -190 & 1 & 0.92 \\
\hline T-GRIP1 & 1070 & 200 & $\mathrm{~N} / \mathrm{A}$ & 200 & $\mathrm{~N} / \mathrm{A}$ & 0.59 & 600 & 670 & 0.81 & 0.83 & -90 & -300 & 0.83 & 0.81 \\
\hline T-GRIP2 & 740 & 500 & $\mathrm{~N} / \mathrm{A}$ & 500 & $\mathrm{~N} / \mathrm{A}$ & 0.58 & 270 & 410 & 0.92 & 0.89 & -50 & -280 & 0.93 & 0.91 \\
\hline L-FORCE1 & 1350 & 1350 & N/A & $\mathrm{N} / \mathrm{A}$ & N/A & $\mathrm{N} / \mathrm{A}$ & 440 & 300 & 0.99 & 0.94 & -20 & -160 & 0.98 & 0.94 \\
\hline T-FORCE1 & 1390 & 1380 & N/A & $\mathrm{N} / \mathrm{A}$ & $\mathrm{N} / \mathrm{A}$ & $\mathrm{N} / \mathrm{A}$ & 720 & 330 & 0.87 & 0.90 & 10 & -320 & 0.83 & 0.85 \\
\hline
\end{tabular}


Table 6

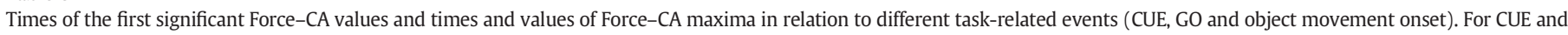

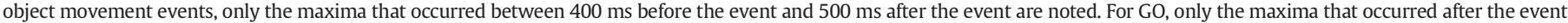
are noted.

\begin{tabular}{|c|c|c|c|c|c|c|c|c|c|c|c|c|c|c|}
\hline \multirow[t]{3}{*}{ Dataset } & \multicolumn{6}{|l|}{ CUE } & \multicolumn{4}{|l|}{ GO } & \multicolumn{4}{|c|}{ Object movement onset } \\
\hline & \multicolumn{2}{|c|}{$\begin{array}{l}\text { Time of first } \\
\text { significant } \\
\text { Force-CA (ms) }\end{array}$} & \multicolumn{2}{|c|}{$\begin{array}{l}\text { Time of } \\
\text { Force-CA } \\
\text { maximum } \\
(\mathrm{ms})\end{array}$} & \multicolumn{2}{|c|}{$\begin{array}{l}\text { Maximum } \\
\text { Force-CA value }\end{array}$} & \multicolumn{2}{|c|}{$\begin{array}{l}\text { Time of } \\
\text { Force-CA } \\
\text { maximum } \\
(\mathrm{ms})\end{array}$} & \multicolumn{2}{|c|}{$\begin{array}{l}\text { Maximum } \\
\text { Force-CA value }\end{array}$} & \multicolumn{2}{|c|}{$\begin{array}{l}\text { Time of Force-CA } \\
\text { maximum (ms) }\end{array}$} & \multicolumn{2}{|c|}{$\begin{array}{l}\text { Maximum } \\
\text { Force-CA value }\end{array}$} \\
\hline & LFC & HFC & LFC & HFC & LFC & HFC & LFC & HFC & LFC & HFC & LFC & HFC & LFC & HFC \\
\hline L-GRIP1 & 1390 & 1430 & $\mathrm{~N} / \mathrm{A}$ & $\mathrm{N} / \mathrm{A}$ & $\mathrm{N} / \mathrm{A}$ & $\mathrm{N} / \mathrm{A}$ & 430 & 400 & 0.80 & 0.64 & 70 & -10 & 0.86 & 0.71 \\
\hline L-GRIP2 & 1480 & 1470 & $\mathrm{~N} / \mathrm{A}$ & N/A & $\mathrm{N} / \mathrm{A}$ & N/A & 430 & 410 & 0.76 & 0.68 & 90 & 0 & 0.82 & 0.76 \\
\hline T-GRIP1 & 1870 & 1850 & $\mathrm{~N} / \mathrm{A}$ & N/A & $\mathrm{N} / \mathrm{A}$ & N/A & 650 & 670 & 0.67 & 0.71 & 70 & -180 & 0.71 & 0.70 \\
\hline T-GRIP2 & 1590 & 1740 & $\mathrm{~N} / \mathrm{A}$ & N/A & $\mathrm{N} / \mathrm{A}$ & N/A & 640 & 710 & 0.69 & 0.68 & 500 & 420 & 0.59 & 0.67 \\
\hline L-FORCE1 & 230 & 250 & 450 & 440 & 0.70 & 0.66 & 650 & 600 & 0.78 & 0.70 & 170 & -60 & 0.86 & 0.79 \\
\hline T-FORCE1 & 470 & 260 & 500 & 420 & 0.59 & 0.67 & 850 & 870 & 0.66 & 0.63 & -10 & -130 & 0.86 & 0.84 \\
\hline
\end{tabular}

cue and is in agreement with other studies that showed changes in spiking activity of PMd neurons on the same time scale (Confais et al., 2012). After becoming significant, Grip-CA increased rapidly to reach a local maximum within $500 \mathrm{~ms}$ after GO. For LFPs aligned to object movement onset, Grip-CA had a broad peak with a maximum around object movement.

Intermediate frequency component (IFC). Similar to Grip-CAs in GRIP datasets, Grip-CAs from an intermediate frequency component in FORCE datasets became significant later, showed lower values and peaked later than Grip-CAs from low and high frequency components. Similarly, Force-CA from an intermediate frequency component was lower than Force-CA from low and high frequency components.

\section{Continuous decoding of grip parameters during the active phase}

In the second step of our analysis we decoded grip parameters from different LFP components and for different temporal offsets. Our intention was to investigate the extent to which it is possible to reconstruct full trajectories of grip parameters from different LFP components (Figs. 7 and 8). Our results show that decoding accuracy, as measured by the execution $F A V$, from low and high frequency components was similar and substantially higher than the execution FAV from the intermediate frequency component (mean execution FAV from a LFP component over all datasets, grip parameters and temporal offsets: LFC: 0.187; IFC: 0.056; HFC: 0.135). Combining the low with the high frequency components led to an $12.8 \%$ improvement in the execution FAV over the higher single component execution $F A V$ (data not shown). Adding the intermediate to either low or high frequency components or to their combination increased the execution FAV by less than $7.4 \%$ of the execution $F A V$ in the absence of intermediate frequency component (mean execution $F A V$ over all datasets, grip parameters and temporal offsets: LFC \& IFC: 0.190; HFC \& IFC: 0.145; LFC \& HFC: 0.211; LFC, IFC \& HFC: 0.212; data not shown).

\section{Evolution of execution FAV for different temporal offsets}

For most combinations of grip parameters and datasets, the execution FAV followed a similar general dependence on the temporal offset
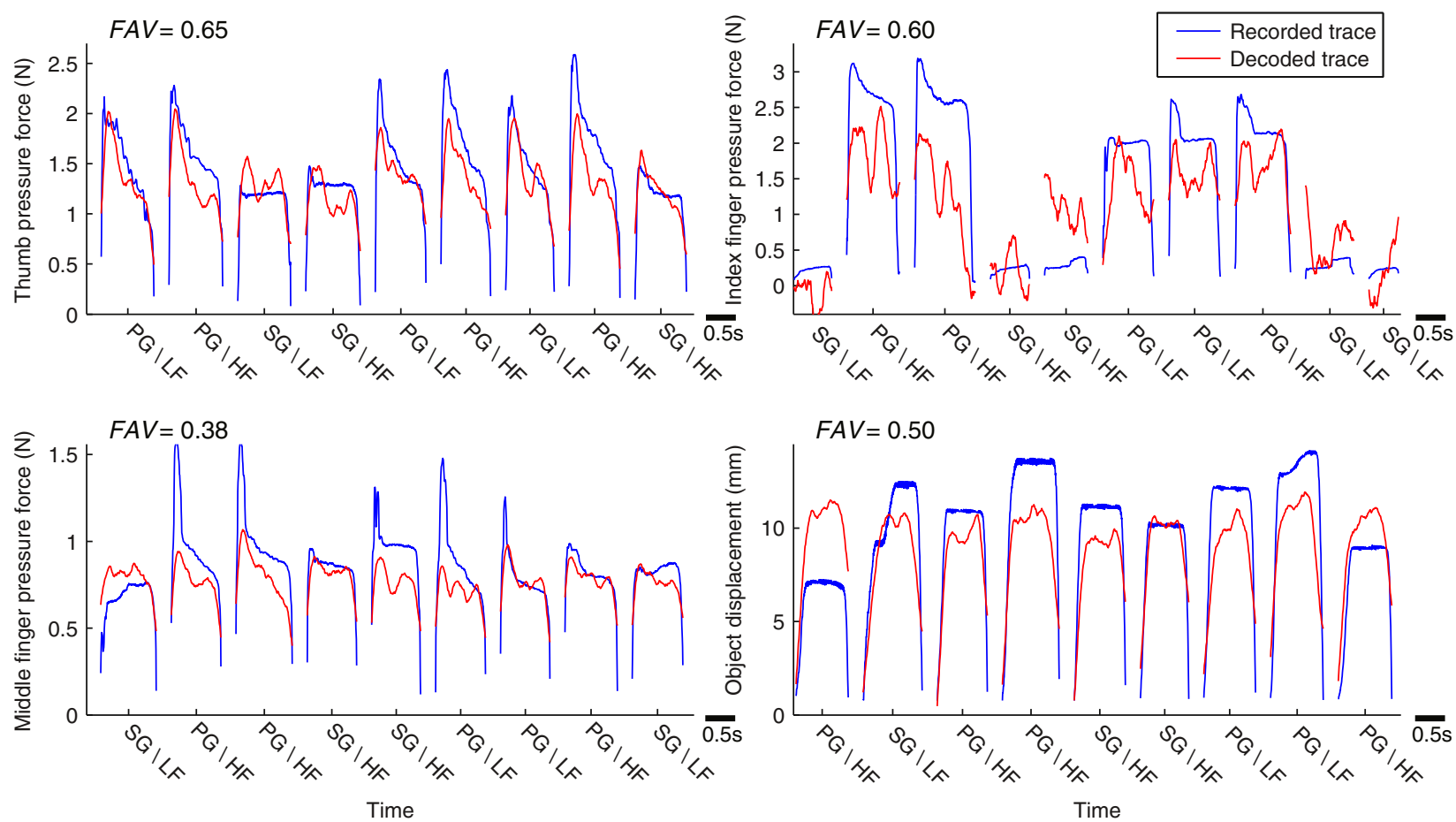

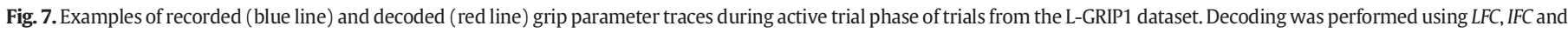

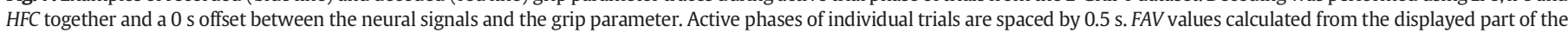
recorded and decoded grip parameter are shown in the top left corner. SG - side grip; PG - precision grip; LF - low force; HF - high force. 

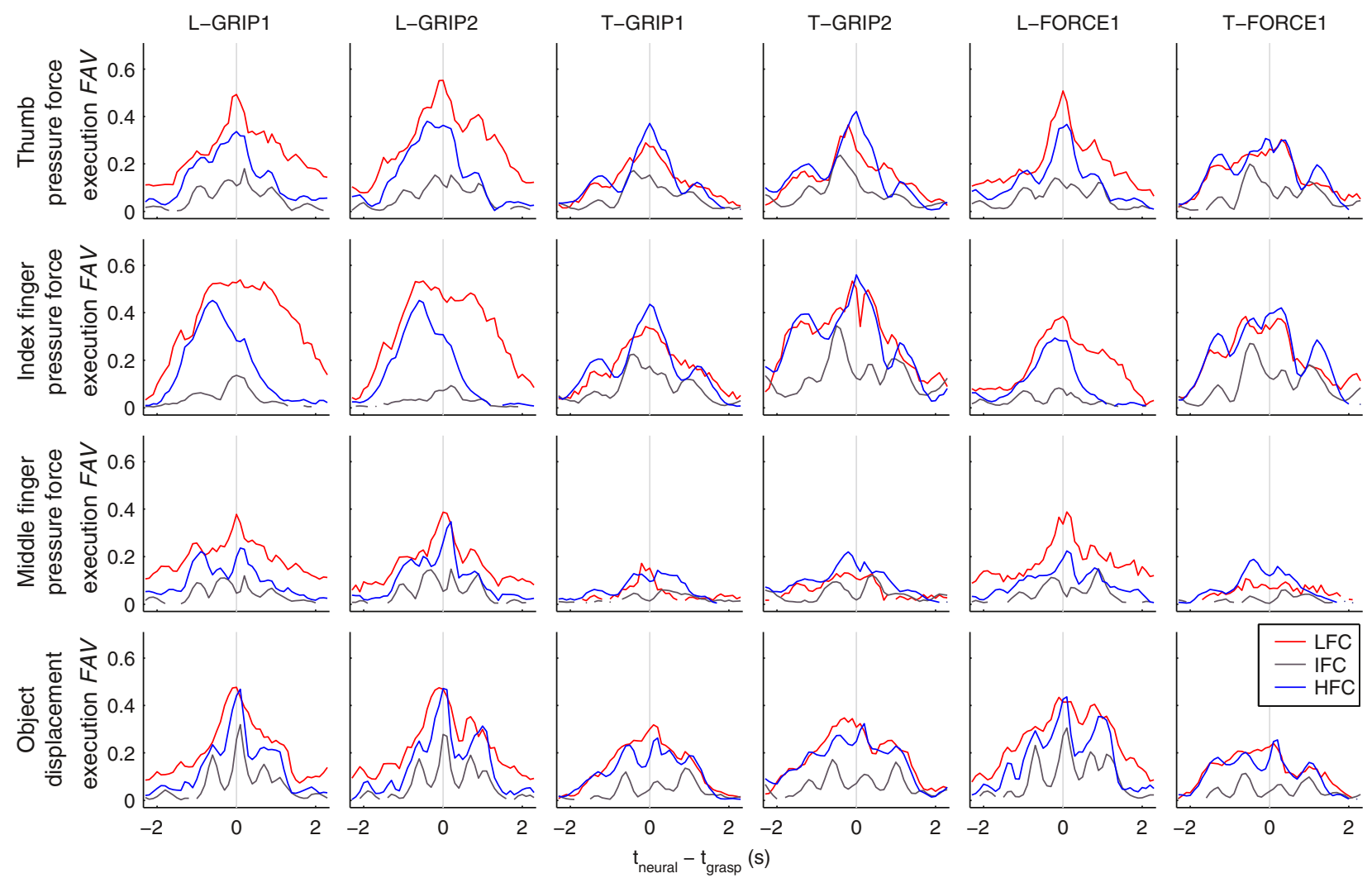

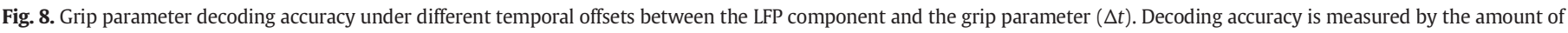

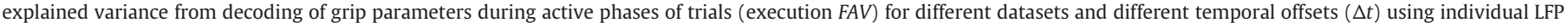
components. Only statistically significant execution $F A V$ values are shown (false discovery rate $<0.01$ ).

$(\Delta t)$ between the decoded grip parameter and the LFPs used for decoding: a slow increase of $F A V$ from the extreme $\Delta t$ values towards 0 with a clear and often broad peak at or around $\Delta t=0$ (Fig. 8). These results may reflect the motor command for the currently exhibited grip parameters and the somatosensory input based on the current grip parameters. For $\Delta t$ of 0 and for using either the low or the high frequency component, thumb and index finger pressure forces can be decoded with similar accuracy, followed by object displacement and middle finger pressure force (mean execution $F A V$ for $\Delta t$ of 0 over all datasets: $L F C$ : thumb: 0.39; index finger: 0.42 ; middle finger: 0.24 ; object displacement: 0.37; HFC: thumb: 0.36; index finger: 0.38; middle finger: 0.17; object displacement: 0.32).

An occasional second execution FAV peak for $\Delta t$ of about $-500 \mathrm{~ms}$ can be seen in Fig. 8, possibly corresponding to the planning of the grip following the CUE. For example, execution $F A V$ for decoding index finger pressure force from high frequency component peaks at $\Delta t$ of $-500 \mathrm{~ms}$ and $-600 \mathrm{~ms}$ in L-GRIP1 and L-GRIP2 datasets (Fig. 8, panels in the second row, first and second columns), respectively. In the same datasets, a small local maximum of execution FAV for decoding index finger pressure force from low frequency component can be seen for $\Delta t$ of $-1400 \mathrm{~ms}$ and $-1500 \mathrm{~ms}$, respectively. Occasionally, another execution $F A V$ peak can be seen for $\Delta t$ of about $1 \mathrm{~s}$ for monkey $L$ and about $1.5 \mathrm{~s}$ for monkey $\mathrm{T}$, possibly reflecting the information encoded in the action of releasing the object.

Continuous decoding of grip parameters during dynamic phase from LFPs recorded during delay period

Results of grip parameter decoding during the active phase (Fig. 8) suggested that LFPs recorded during the delay period of the trial may be used to continuously decode parameters of grip movements that monkeys executed after the GO. However, due to the broad central peak of the autocorrelation of the grip parameter traces and the variability of the length of the active phases, it was not possible to completely disambiguate the execution FAV contributions of the LFPs recorded during the delay period, i.e. corresponding to movement planning, and the LFPs recorded after GO, i.e. corresponding to movement execution. To further investigate the amount of information related to movement execution available in LFPs from the delay period, we decoded only the dynamic phase of the trial, which was short and has a narrow central peak of the autocorrelation, from LFPs recorded only during the delay period and measured the decoding accuracy using planning FAV (Fig. 9). The decoding was performed for different temporal offsets $(\tau)$ of the LFPs in relation to CUE.

In the GRIP datasets, the dependence of the planning FAV in the low and high frequency components on $\tau$ exhibits two distinctive peaks. The first peak occurs for $\tau<400 \mathrm{~ms}$, i.e. when the dynamic phase of the grip parameter was decoded from LFPs recorded at the beginning of the delay period. The second peak appears for $\tau>950 \mathrm{~ms}$, i.e. when using the LFPs recorded at the end of the delay period, just before GO to decode the dynamic phase of the grip parameters. The two peaks can be clearly seen for decoding the thumb and index finger pressure forces in the GRIP datasets for monkey L (Fig. 9, panels in the first and the second rows of the first and the second columns). Maxima planning FAV of the first peak ranged between 0.07 and 0.45 and occurred for a $\tau$ of $60 \mathrm{~ms}$ to $240 \mathrm{~ms}$ when calculated from the low frequency component and for a $\tau$ of $150 \mathrm{~ms}$ to $360 \mathrm{~ms}$ when calculated from the high frequency component. The second planning FAV peak occurred at a $\tau$ of $990 \mathrm{~ms}$ to $1200 \mathrm{~ms}$. In GRIP datasets for monkey T, the planning FAV was substantially lower when compared to that of monkey L. In some cases, one of the two peaks did not occur. For example, in the T-GRIP1 dataset, only the second planning FAV peak calculated from low frequency 


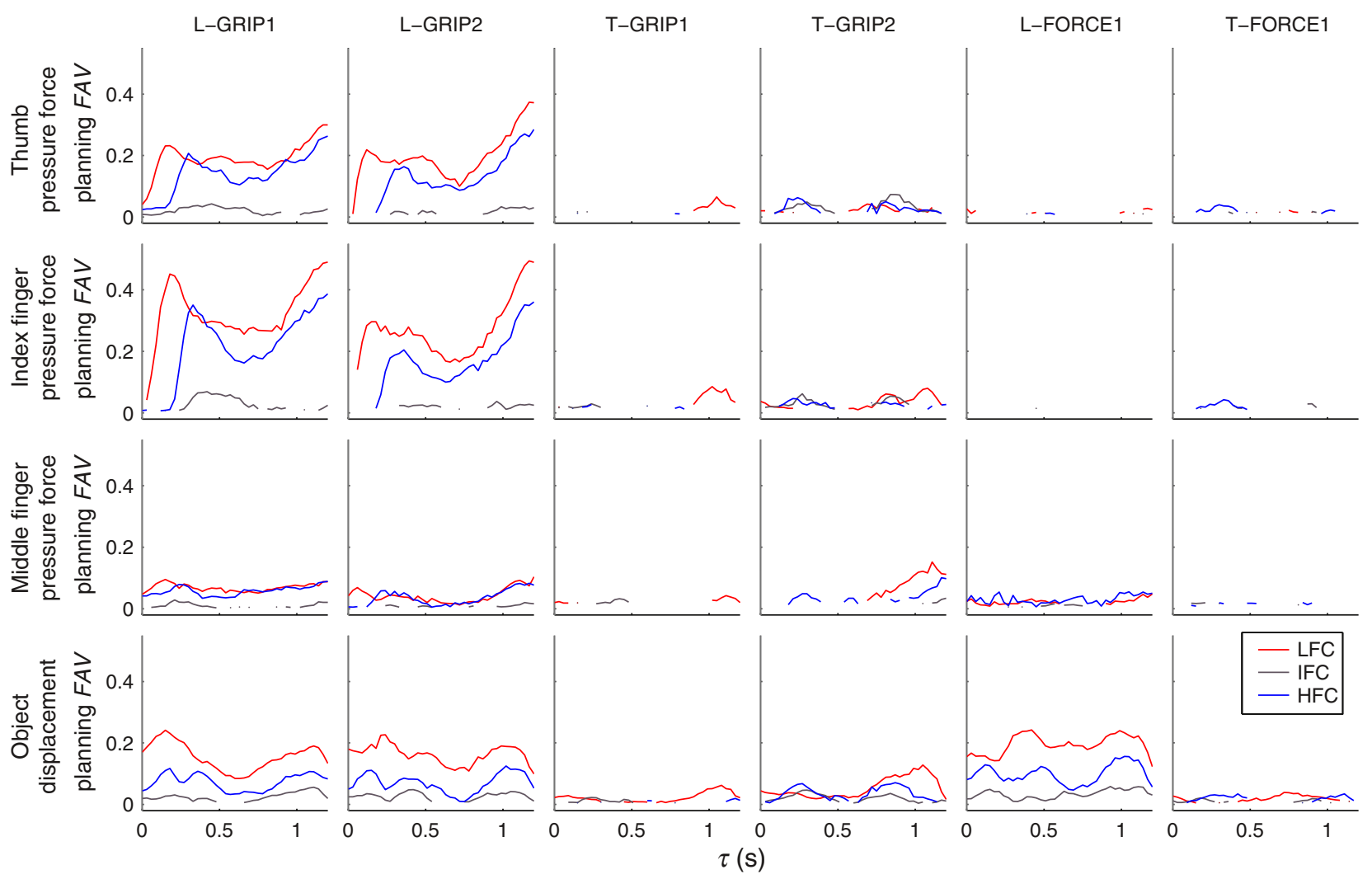

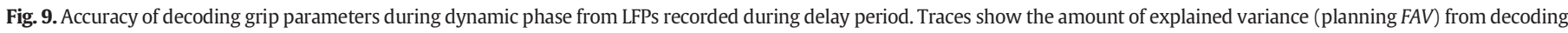

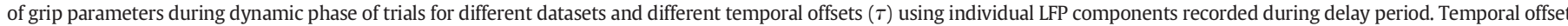

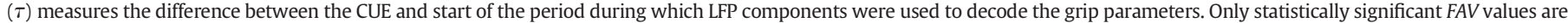
shown (false discovery rate $<0.01$ ).

component was clearly visible. These results suggest that monkey T may not have planned his grip movements during the delay period in the majority of the trials.

In the FORCE datasets of both monkeys (Fig. 9, two right most columns), thumb, index finger and middle finger pressure force planning $F A V$ were below 0.06 and, for majority of $\tau$, were not significant. This implies that, during FORCE sessions, the monkeys did not plan finger pressure forces during the delay period, even though some of the load force information was encoded in the LFPs (Fig. 5). On the other hand, object displacement planning FAV calculated from the L-FORCE1 dataset was substantially higher than the object displacement planning FAV calculated from the T-FORCE1 dataset, implying that monkey $L$ still partially planned his grip movements during the delay period based on the information provided by the CUE in the FORCE task, whereas monkey T may not have done so.

When decoding object displacement, planning FAV peaks were broader and much less pronounced compared to finger pressure forces, probably because the object displacement does not depend on the information provided by the CUE and because the dynamic phase of the object displacement usually lasts longer than the dynamic phase of finger pressure forces and has a higher autocorrelation.

\section{Discussion}

We investigated the extent to which LFP modulations recorded from motor cortex of macaque monkeys contain information about intended grasping movements, especially kinetic parameters, before and during their execution. The object was grasped with either a precision or a side grip, and under low or high load force. Our findings demonstrate that the grip type as well as the load forces can be classified from the low and high frequency components of the LFP with substantial accuracy. In addition, successful classification can be achieved long before the initiation of the grasping movement. Furthermore, we show that the finger pressure forces applied on the object during movement execution can be continuously decoded from LFPs recorded during the execution of the movement. Intended pressure forces can also be decoded from LFPs recorded exclusively before the initiation of the movement.

\section{Similarity of identified LFP components to previously described LFP bands}

We identified three distinctive LFP components that modulated during execution of reach and grasp movements. The low frequency component $(L F C)$ was set a priori to be the band-pass filtered signal $(0.3 \mathrm{~Hz}-7 \mathrm{~Hz})$, while intermediate and high frequency components (IFC and HFC) were identified using a procedure that maximizes the signal-to-noise ratio of a continuous band (Fig. 3; Milekovic et al., 2012). In our recordings, LFC modulated during movement execution similar to the previously described LFP modulations in a similar frequency range (Mehring et al., 2003; Mollazadeh et al., 2011; Rickert et al., 2005; Riehle et al., 2013). The IFC frequency bands (monkey L: 13-43 Hz; monkey T: $10-40 \mathrm{~Hz}$ ) contained the beta frequency band (10-30 Hz), and its modulations (Figs. 3 and 4) were similar to amplitude modulations observed in this band for LFPs and human EEGs during hand movements (Baker et al., 1997; Kilavik et al., 2013; Spinks et al., 2008; Zaepffel et al., 2013). Bands chosen here were slightly wider either due to differences in individual monkeys (Kilavik et al., 2012) or due to a different method of selection. HFC frequency bands (monkey L: 79-250 Hz; monkey T: 82-244 Hz) overlapped with the band usually referred to as high-gamma LFPs. In our study, HFC increases from baseline during hand movements, similar to high-gamma 
LFP amplitude movement-related modulations reported elsewhere (Mollazadeh et al., 2011; Rickert et al., 2005).

\section{Grip parameter related information content of LFP components}

All three identified LFP components have previously been found to contain information about voluntary movements in primates (Bansal et al., 2012; Zhuang et al., 2010). Here, we show that all three components contain information about the intended grip type and load force before and during movement execution. LFC and HFC contain similar amounts of information, whereas the amount of information in IFC is substantially smaller, as shown by substantially lower grip type classification accuracy (Grip-CA) and load force classification accuracy (ForceCA; Figs. 5 and 6), as well as substantially lower accuracy of decoding grip parameters, as measured by planning and execution FAV (Figs. 8 and 9). Combining LFC and HFC improves the decoding accuracy slightly, indicating that the information contained in these two components is not completely redundant. Adding IFC to either the combination of $L F C$ and HFC or to each separately leads to only a small improvement. Recent studies showed that action potentials of neurons in the vicinity of recording electrodes can contribute to LFPs (Buzsaki et al., 2012) even just above tens of $\mathrm{Hz}$ (Reimann et al., 2013; Waldert et al., 2013). Thus, grip type and load force information present in IFC and HFC may have partially come from action potentials, potentially making the high and intermediate frequency LFPs redundant if action potentials can be successfully extracted from the neural recordings. However, LFC is considered to predominantly reflect the dendritic currents, and contamination from action potentials is considered unrealistic (Waldert et al., 2013). Thus, our results indicate that LFPs may contain information not present in action potentials.

Maximum Grip-CA values from LFC of between $83 \%$ and $100 \%$ obtained in our study match those obtained by Mollazadeh et al. (2011). They also demonstrated substantially lower classification accuracy of grip types when using LFP high gamma band $(100-170 \mathrm{~Hz})$ spectral power as classification features. Our Grip-CA values from HFC were similar to those from $L F C$ and substantially higher than those of Mollazadeh et al. (2011). The seeming disparity may have been caused by the narrower frequency band used by Mollazadeh et al. (2011) that may have failed to capture all grip-related LFP modulation. In our study, the HFC frequency band was selected based on the properties of the signal, which may have served as a better decoding signal. On the other hand, Mollazadeh et al. (2011) attempted to classify more grip types, which made the task harder and, thus, may have contributed to the difference. Grip-CA values obtained from human ECoG (Pistohl et al., 2012) showed similar temporal evolution around the object movement, but were substantially lower (maximum Grip-CA of 0.81 obtained from $L F C$ ). When decoding pressure force on the grasped object, mean $F A V$ value obtained from human ECoG was 0.6 (Flint et al., 2014), similar to that obtained from monkey ECoG (Chen et al., 2014). We decoded pressure forces of individual fingers and reached lower $F A V$ values on average. However, decoding of three degrees of freedom (three fingers) as opposed to one (pressure force on the object) made our task more complex, which may have led to lower FAV.

After reaching the object, monkeys gripped and pulled the object at the same time. Thus, LFP modulations that followed the object movement reflect kinetics and kinematics of both pulling and gripping. However, pulling kinematics, measured by object displacement, were similar for all tasks that the monkeys performed and differed only slightly for different load forces (Fig. 2). Since the object movement was frictionless, the pulling kinetics was proportional to the second derivative of the kinematics and, thus, also similar between tasks. Small differences in the pulling kinematics compared to differences in finger pressure kinetics likely led to small differences in the LFP modulations and, thus, led to only small Grip-CA contributions and slightly higher Force-CA contributions.
Temporal evolution of information related to grip parameters during the delay period

In GRIP datasets, grip type classification accuracies increased towards the end of the delay period, i.e. in expectation of GO. Furthermore, our ability to decode executed grip parameters increased when using LFPs recorded at the end of the delay period. Thus, in expectation of the GO, the monkeys may have recalled the previously made movement plan or, especially in the case of monkey T for GRIP datasets, generated the movement plan based on the memorized information provided by the CUE.

In FORCE datasets, the load force classification accuracy during the delay period indicates that the motor cortex takes part in planning the load force. However, in our task, finger pressure forces do not depend on load force as much as on the grip type (Fig. 2). Thus, it is likely that the load force information could hardly be used to plan the force since the finger positions used to grasp the object were not yet known. Furthermore, monkeys could adapt to the load force during the grasp and get the reward, even in the case of incorrectly estimated load force based on information provided by CUE or GO. Altogether, this would explain the substantially lower load force classification accuracies in the FORCE task than the grip type classification accuracies in the GRIP task during the delay period.

For monkey L, we obtained clear peaks of grip type (GRIP task) and load force (FORCE task) classification accuracies within $500 \mathrm{~ms}$ after presenting task-relevant information (CUE). Furthermore, we were able to decode the dynamic phase of the executed thumb and index finger pressure forces from the LFPs recorded at the beginning of the delay period in the GRIP task (Fig. 9). These results suggest that monkey L planned the thumb and index finger pressure forces already at the beginning of the delay period and that this process was reflected in motor cortical LFPs. However, in the same period, the planning FAV related to the middle finger pressure force was substantially lower. This effect may be due to the fact that the force applied by the middle finger was roughly similar during precision and side grips and did not have to be planned in advance by the monkey. Also, we cannot exclude that at this location in the motor cortex, neural activity was more related to thumb and index finger pressure forces than related to the middle finger pressure forces. The same classification peak was absent and the planning FAV was low in monkey T GRIP datasets, which suggests that this monkey may not have planned grasping movements in the majority of the trials. This conclusion is further supported by longer times required for monkey $\mathrm{T}$ to initiate the object movement following GO.

While the grip related information present in LFPs during the delay period may completely or partially reflect the movement plan, other processes may have contributed to it. Monkeys may have shifted their posture, modified their hand position or muscle tension in a taskdependent fashion, and such changes in the behavior may have been reflected in the LFPs. We did not monitor the muscle activity or the arm kinematics to exclude these possibilities. On the other hand, absence of the high Grip-CA and Force-CA values during the delay period and the low planning FAV do not necessarily imply the absence of a movement plan. The quality of the recordings can be variable and may contain different levels of noise. In the case of monkey T, LFPs may have been more noisy and recorded from a cortical location that was less related to movement planning, thus inducing lower CA and FAV values regardless of whether or not the animal planned the movement.

\section{Anatomical location of implanted arrays}

Microelectrode arrays were implanted on the surface of the precentral gyrus in the location that partially covered areas MI and PMd. Our ability to classify grip types and decode grip parameters from LFPs recorded during the delay period may be the consequence of recordings originating from the premotor cortex, thought to be responsible for movement planning (Shenoy et al., 2011). The lack of 
clear and precise observable anatomical borders of MI and PMd on the cortical surface during surgery prevented us from distinguishing between LFPs originating from these two areas.

The area of the motor cortex we recorded from does not have direct connections to the motoneurons. It only has indirect connections to the spinal cord circuitry through spinal interneurons (Rathelot and Strick, 2009). Thus, recordings from the caudal sulcus of MI, where layer 5 neurons with direct connections to motoneurons are located (Rathelot and Strick, 2009) and force information is clearly represented (Bennett and Lemon, 1994; Maier et al., 1993), may improve the ability to decode grip parameters during movement execution. However, placing the type of microelectrode array used in our study inside the sulcus is not yet possible.

\section{Relevance for clinical applications}

Brain-machine interfaces (BMIs) are investigational devices that are being developed to provide paralyzed individuals with the ability to control natural or external effectors, such as a robotic arm, using their neural activity (Donoghue et al., 2007; Hochberg, 2008). Historically, BMI approaches have focused on the kinematic control of reach movements, while specification of kinetic parameters, such as the force used to grasp an object, has been relegated to automated control or to the experimenter (Collinger et al., 2013; Hochberg et al., 2012; Velliste et al., 2008). However, the use of kinetic control may substantially improve BMIs designed to enable users to grasp and manipulate objects. For example, decoding finger pressure forces may enable users of BMI controlled prosthetic arms with the ability to hold fragile objects, such as plastic cups or biscuits, without crushing them. In this study, we showed that finger pressure forces can be decoded from LFPs recorded in non-human primate motor cortex, which may enable BMI driven arm prosthesis with precise finger pressure force control during object grasping and manipulation. Here, the demonstrated decoding of intended finger-pressure forces and hand positions in advance of the execution of a grasping movement could allow prosthetic arms to calibrate the grip in advance of object contact, thus increasing the possibility that the grasp will be successful.

Previous studies demonstrated that the use of more complex algorithms may lead to higher classification and decoding accuracies (Aydemir and Kayikcioglu, 2014; Garrett et al., 2003; Kim et al., 2006, 2008; Ku et al., 2008). We opted to use a linear classifier and decoder, which offer a transparent way to follow temporal evolution of information investigated here. Thus, in future studies, it may be possible to improve the classification and decoding accuracies beyond those achieved in our study to further benefit BMI applications.

\section{Acknowledgments}

This work was supported by the German Federal Ministry of Education and Research (BMBF) grant 01GQ0830 to BFNT Freiburg and Tübingen, Imperial College London, IBRO InEurope Short Stay Grant program, RIKEN-CNRS Collaborative Research Agreement, ANR GRASP (France), BrainScaleS (EU Grant 269912), Helmoltz portfolio theme "Supercomputing and modeling for the human brain" (SMHB), U.S. National Institute of Neurological Disorders and Stroke (NINDS) K01 Career Award NS057389, and the Pablo J. Salame '88 Goldman Sachs endowed Professorship in Computational Neuroscience, Brown University.

\section{Appendix A. Supplementary data}

Supplementary data to this article can be found online at http://dx. doi.org/10.1016/j.neuroimage.2015.04.008.

\section{References}

Aydemir, O., Kayikcioglu, T., 2014. Decision tree structure based classification of EEC signals recorded during two dimensional cursor movement imagery. J. Neurosci. Methods 229, 68-75.

Baker, S.N., Olivier, E., Lemon, R.N., 1997. Coherent oscillations in monkey motor cortex and hand muscle EMG show task-dependent modulation. J. Physiol. Lond. 501, 225-241.

Ball, T., Schulze-Bonhage, A., Aertsen, A., Mehring, C., 2009. Differential representation of arm movement direction in relation to cortical anatomy and function. J. Neural Eng. 6 016006.

Bansal, A.K., Truccolo, W., Vargas-Irwin, C.E., Donoghue, J.P., 2012. Decoding 3D reach and grasp from hybrid signals in motor and premotor cortices: spikes, multiunit activity, and local field potentials. J. Neurophysiol. 107, 1337-1355.

Barnard, G.A., 1945. A new test for $2 \times 2$ tables. Nature 156, 783-784.

Benjamini, Y., Hochberg, Y., 1995. Controlling the false discovery rate - a practical and powerful approach to multiple testing. J. R. Stat. Soc. Ser. B Methodol. 57, 289-300.

Benjamini, Y., Yekutieli, D., 2001. The control of the false discovery rate in multiple testing under dependency. J. R. Stat. Soc. Ser. B Methodol. 29, 1165-1188.

Bennett, K.M., Lemon, R.N., 1994. The influence of single monkey cortico-motoneurona cells at different levels of activity in target muscles. J. Physiol. 477 (Pt 2), 291-307.

Buzsaki, G., Anastassiou, C.A., Koch, C., 2012. The origin of extracellular fields and currents-EEG, ECoG, LFP and spikes. Nat. Rev. Neurosci. 13, 407-420.

Carpaneto, J., Umilta, M.A., Fogassi, L., Murata, A., Gallese, V., Micera, S., Raos, V., 2011. Decoding the activity of grasping neurons recorded from the ventral premotor area F5 of the macaque monkey. Neuroscience 188, 80-94.

Chen, C., Shin, D., Watanabe, H., Nakanishi, Y., Kambara, H., Yoshimura, N., Nambu, A., Isa, T., Nishimura, Y., Koike, Y., 2014. Decoding grasp force profile from electrocorticography signals in non-human primate sensorimotor cortex. Neurosci. Res. 83, 1-7.

Collinger, J.L., Wodlinger, B., Downey, J.E., Wang, W., Tyler-Kabara, E.C., Weber, D.J., McMorland, A.J., Velliste, M., Boninger, M.L., Schwartz, A.B., 2013. High-performance neuroprosthetic control by an individual with tetraplegia. Lancet 381, 557-564.

Confais, J., Kilavik, B.E., Ponce-Alvarez, A., Riehle, A., 2012. On the anticipatory precue activity in motor cortex. J. Neurosci. 32, 15359-15368.

Donoghue, J.P., Nurmikko, A., Black, M., Hochberg, L.R., 2007. Assistive technology and robotic control using motor cortex ensemble-based neural interface systems in humans with tetraplegia. J. Physiol. Lond. 579, 603-611.

Fagg, A.H., Ojakangas, G.W., Miller, L.E., Hatsopoulos, N.G., 2009. Kinetic trajectory decoding using motor cortical ensembles. IEEE Trans. Neural Syst. Rehabil. Eng. 17, 487-496.

Fisher, R.A., 1922. On the interpretation of $\mathrm{x}(2)$ from contingency tables, and the calculation of P. Journal of the Royal Statistical Society 85, 87-94.

Flint, R.D., Ethier, C., Oby, E.R., Miller, L.E., Slutzky, M.W., 2012a. Local field potentials allow accurate decoding of muscle activity. J. Neurophysiol. 108, 18-24.

Flint, R.D., Lindberg, E.W., Jordan, L.R., Miller, L.E., Slutzky, M.W., 2012b. Accurate decoding of reaching movements from field potentials in the absence of spikes. J. Neural Eng. 9, 046006.

Flint, R.D., Wang, P.T., Wright, Z.A., King, C.E., Krucoff, M.O., Schuele, S.U., Rosenow, J.M., Hsu, F.P., Liu, C.Y., Lin, J.J., Sazgar, M., Millett, D.E., Shaw, S.J., Nenadic, Z., Do, A.H., Slutzky, M.W., 2014. Extracting kinetic information from human motor cortical signals. NeuroImage 101, 695-703.

Friedman, J.H., 1989. Regularized discriminant-analysis. J. Am. Stat. Assoc. 84, 165-175.

Garrett, D., Peterson, D.A., Anderson, C.W., Thaut, M.H., 2003. Comparison of linear, nonlinear, and feature selection methods for EEG signal classification. IEEE Trans. Neural Syst. Rehabil. Eng. 11, 141-144.

Hammer, J., Fischer, J., Ruescher, J., Schulze-Bonhage, A., Aertsen, A., Ball, T., 2013. The role of ECoG magnitude and phase in decoding position, velocity, and acceleration during continuous motor behavior. Front. Neurosci. 7, 200.

Hastie, T., Tibshirani, R., Friedman, J.H., 2009. The Elements of Statistical Learning: Data Mining, Inference, and Prediction. 2nd ed. Springer, New York.

Hendrix, C.M., Mason, C.R., Ebner, T.J., 2009. Signaling of grasp dimension and grasp force in dorsal premotor cortex and primary motor cortex neurons during reach to grasp in the monkey. J. Neurophysiol. 102, 132-145.

Hochberg, L.R., 2008. Turning thought into action. N. Engl. J. Med. 359, 1175-1177.

Hochberg, L.R., Bacher, D., Jarosiewicz, B., Masse, N.Y., Simeral, J.D., Vogel, J., Haddadin, S., Liu, J., Cash, S.S., van der Smagt, P., Donoghue, J.P., 2012. Reach and grasp by people with tetraplegia using a neurally controlled robotic arm. Nature 485, 372-375.

Kilavik, B.E., Ponce-Alvarez, A., Trachel, R., Confais, J., Takerkart, S., Riehle, A., 2012. Context-related frequency modulations of macaque motor cortical LFP beta oscillations. Cereb. Cortex 22, 2148-2159.

Kilavik, B.E., Zaepffel, M., Brovelli, A., MacKay, W.A., Riehle, A., 2013. The ups and downs of beta oscillations in sensorimotor cortex. Exp. Neurol. 245, 15-26.

Kim, S.P., Sanchez, J.C., Rao, Y.N., Erdogmus, D., Carmena, J.M., Lebedev, M.A., Nicolelis, M.A., Principe, J.C., 2006. A comparison of optimal MIMO linear and nonlinear models for brain-machine interfaces. J. Neural Eng. 3, 145-161.

Kim, S.P., Simeral, J.D., Hochberg, L.R., Donoghue, J.P., Black, M.J., 2008. Neural control of computer cursor velocity by decoding motor cortical spiking activity in humans with tetraplegia. J. Neural Eng. 5, 455-476.

Ku, S.P., Gretton, A., Macke, J., Logothetis, N.K., 2008. Comparison of pattern recognition methods in classifying high-resolution BOLD signals obtained at high magnetic field in monkeys. Magn. Reson. Imaging 26, 1007-1014.

Kubanek, J., Miller, K.J., Ojemann, J.G., Wolpaw, J.R., Schalk, G., 2009. Decoding flexion of individual fingers using electrocorticographic signals in humans. J. Neural Eng. 6, 066001.

Leski, S., Linden, H., Tetzlaff, T., Pettersen, K.H., Einevoll, G.T., 2013. Frequency dependence of signal power and spatial reach of the local field potential. PLoS Comput. Biol. 9, e1003137. 
Li, Y., Hao, Y., Wang, D., Zhang, Q., Liao, Y., Zheng, X., Chen, W., 2012. Decoding grasp types with high frequency of local field potentials from primate primary dorsal premotor cortex. Conf. Proc. IEEE Eng. Med. Biol. Soc. 2012, 1691-1694.

Logothetis, N.K., Kayser, C., Oeltermann, A., 2007. In vivo measurement of cortical impedance spectrum in monkeys: implications for signal propagation. Neuron 55, 809-823.

Maier, M.A., Bennett, K.M., Hepp-Reymond, M.C., Lemon, R.N., 1993. Contribution of the monkey corticomotoneuronal system to the control of force in precision grip. J. Neurophysiol. 69, 772-785.

Markowitz, D.A., Wong, Y.T., Gray, C.M., Pesaran, B., 2011. Optimizing the decoding of movement goals from local field potentials in macaque cortex. J. Neurosci. 31, 18412-18422.

Mehring, C., Rickert, J., Vaadia, E., Cardosa de Oliveira, S., Aertsen, A., Rotter, S., 2003. Inference of hand movements from local field potentials in monkey motor cortex. Nat. Neurosci. 6, 1253-1254.

Milekovic, T., Ball, T., Schulze-Bonhage, A Aertsen, A., Mehring C., 2012. Error-related electrocorticographic activity in humans during continuous movements. J. Neural Eng. 9, 026007.

Milekovic, T., Ball, T., Schulze-Bonhage, A., Aertsen, A., Mehring, C., 2013. Detection of error related neuronal responses recorded by electrocorticography in humans during continuous movements. PLoS One 8, e55235.

Miller, K.J., 2010. Broadband spectral change: evidence for a macroscale correlate of population firing rate? J. Neurosci. 30, 6477-6479.

Miller, K.J., Sorensen, L.B., Ojemann, J.G., den Nijs, M., 2009. Power-law scaling in the brain surface electric potential. PLoS Comput. Biol. 5, e1000609.

Mitzdorf, U., 1985. Current source-density method and application in cat cerebral cortex: investigation of evoked potentials and EEG phenomena. Physiol. Rev. 65, 37-100.

Mollazadeh, M., Aggarwal, V., Davidson, A.G., Law, A.J., Thakor, N.V., Schieber, M.H., 2011 Spatiotemporal variation of multiple neurophysiological signals in the primary motor cortex during dexterous reach-to-grasp movements. J. Neurosci. 31, 15531-15543.

Pistohl, T., Schulze-Bonhage, A., Aertsen, A., Mehring, C., Ball, T., 2012. Decoding natural grasp types from human ECoG. Neurolmage 59, 248-260.

Rathelot, J.A., Strick, P.L., 2009. Subdivisions of primary motor cortex based on corticomotoneuronal cells. Proc. Natl. Acad. Sci. U. S. A. 106, 918-923.

Reimann, M.W., Anastassiou, C.A., Perin, R., Hill, S.L., Markram, H., Koch, C., 2013. A biophysically detailed model of neocortical local field potentials predicts the critical role of active membrane currents. Neuron 79, 375-390.

Rickert, J., Oliveira, S.C., Vaadia, E., Aertsen, A., Rotter, S., Mehring, C., 2005. Encoding of movement direction in different frequency ranges of motor cortical local field potentials. J. Neurosci. 25, 8815-8824.
Riehle, A., Wirtssohn, S., Grün, S., Brochier, T., 2013. Mapping the spatio-temporal structure of motor cortical LFP and spiking activities during reach-to-grasp movements. Front. Neural Circ. 7, 48.

Saleh, M., Takahashi, K., Hatsopoulos, N.G., 2012. Encoding of coordinated reach and grasp trajectories in primary motor cortex. J. Neurosci. 32, 1220-1232.

Santhanam, G., Ryu, S.I., Yu, B.M., Afshar, A., Shenoy, K.V., 2006. A high-performance brain-computer interface. Nature 442, 195-198.

Savitzky, A., Golay, M.J.E., 1964. Smoothing and differentiation of data by simplified least squares procedures. Anal. Chem. 36, 1627-1639.

Schieber, M.H., Poliakov, A.V., 1998. Partial inactivation of the primary motor cortex hand area: effects on individuated finger movements. J. Neurosci. 18, 9038-9054.

Shenoy, K.V., Kaufman, M.T., Sahani, M., Churchland, M.M., 2011. A dynamical systems view of motor preparation: implications for neural prosthetic system design. Prog. Brain Res. 192, 33-58.

Spinks, R.L. Kraskov, A., Brochier, T., Umilta, M.A., Lemon, R.N., 2008. Selectivity for grasp in local field potential and single neuron activity recorded simultaneously from M1 and F5 in the awake macaque monkey. J. Neurosci. 28, 10961-10971.

Steinier, J., Termonia, Y., Deltour, J., 1972. Smoothing and differentiation of data by simplified least square procedure. Anal. Chem. 44, 1906-1909.

Townsend, B.R., Subasi, E., Scherberger, H., 2011. Grasp movement decoding from premotor and parietal cortex. J. Neurosci. 31, 14386-14398.

Vargas-Irwin, C.E., Shakhnarovich, G., Yadollahpour, P., Mislow, J.M., Black, M.J., Donoghue, J.P., 2010. Decoding complete reach and grasp actions from local primary motor cortex populations. J. Neurosci. 30, 9659-9669.

Velliste, M., Perel, S., Spalding, C., Whitford, A., Schwartz, A., 2008. Cortical control of a prosthetic arm for self-feeding. Nature 453, 1098-1101.

Waldert, S., Lemon, R.N., Kraskov, A., 2013. Influence of spiking activity on cortical local field potentials. J. Physiol. 591, 5291-5303.

Xing, D., Yeh, C.I., Shapley, R.M., 2009. Spatial spread of the local field potential and its laminar variation in visual cortex. J. Neurosci. 29, 11540-11549.

Zaepffel, M., Trachel, R., Kilavik, B.E., Brochier, T., 2013. Modulations of EEG beta power during planning and execution of grasping movements. PLoS One 8, e60060.

Zhuang, J., Truccolo, W., Vargas-Irwin, C., Donoghue, J.P., 2010. Decoding 3-D reach and grasp kinematics from high-frequency local field potentials in primate primary motor cortex. IEEE Trans. Biomed. Eng. 57, 1774-1784. 